\title{
1 Transcriptional activation of auxin biosynthesis drives developmental reprogramming of \\ 2 differentiated cells
}

3

4 Yuki Sakamoto $^{1,2}$, Ayako Kawamura ${ }^{2}$, Takamasa Suzuki $^{3}$, Shoji Segami $^{4,5}$, Masayoshi Maeshima ${ }^{3}$,

5 Stefanie Polyn ${ }^{6,7}$, Lieven De Veylder ${ }^{6,7}$, Keiko Sugimoto ${ }^{1,2}$.

6

$7 \quad{ }^{1}$ Department of Biological Sciences, Graduate School of Science, The University of Tokyo, 7-3-1

8 Hongo, Bunkyo-ku, Tokyo 113-0033, Japan.

$9{ }^{2}$ Center for Sustainable Resource Science, RIKEN, 1-7-22 Suehiro-cho, Tsurumi, Yokohama, 10 Kanagawa 230-0045, Japan.

$11{ }^{3}$ Department of Biological Chemistry, College of Bioscience and Biotechnology, Chubu University, 121200 Matsumoto-cho, Kasugai, Aichi 487-8501, Japan.

$13{ }^{4}$ Division of Evolutionary Biology, National Institute for Basic Biology, Okazaki, Aichi 444-8585, 14 Japan.

$15{ }^{5}$ Department of Basic Biology, School of Life Science, The Graduate University for Advanced 16 Studies, SOKENDAI, Okazaki, Aichi 444-8585, Japan.

$17{ }^{6}$ Department of Plant Biotechnology and Bioinformatics, Ghent University, Ghent B-9052, Belgium.

$18{ }^{7}$ VIB Center for Plant Systems Biology, Ghent B-9052, Belgium. 


\section{Abstract}

23 Plant cells exhibit remarkable plasticity of their differentiation states, enabling regeneration

24 of whole plants from differentiated somatic cells. How they revert cell fate and express pluripotency,

25 however, remains unclear. Here we show that transcriptional activation of auxin biosynthesis is

26 crucial for reprogramming differentiated Arabidopsis leaf cells. We demonstrate that interfering with

27 the activity of histone acetyltransferases dramatically reduces callus formation from leaf mesophyll

28 protoplasts. Impaired histone acetylation predominantly affects transcription of auxin biosynthesis

29 genes. Auxin biosynthesis is in turn required to accomplish initial cell division through the activation

30 of G2/M phase genes mediated by MYB DOMAIN PROTEIN 3-RELATED (MYB3Rs). We further

31 show that the AUXIN RESPONSE FACTOR 7 (ARF7)/ARF19 and INDOLE-3-ACETIC ACID

32 INDUCIBLE 3 (IAA3)/IAA18-mediated auxin signaling pathway is responsible for cell cycle

33 reactivation in protoplasts. These findings provide novel mechanistic model of how differentiated

34 plant cells can revert their fate and reinitiate the cell cycle to become pluripotent. 
Tight coordination of cell proliferation and differentiation is central to optimize plant organ

37 development. Typically, during a normal developmental program, meristematic cells continue to

38 proliferate until they start to differentiate, and once differentiated, the somatic cells usually remain

39 mitotically inactivate ${ }^{1-3}$. Such a relationship between mitosis and cellular differentiation status should

40 be also critical during developmental reprogramming through which plant somatic cells convert their

41 fates and regenerate new tissues or organs. Previous studies indeed suggest that reactivation of

42 somatic cell division contributes to cell fate conversion and acquisition of pluripotency presumably

43 by helping cells dilute existing identity and activate a new developmental program ${ }^{4-6}$. Uncovering

44 how differentiated cells reinitiate cell division is therefore crucial to understand how somatic cells

45 initiate reprogramming.

46 Mesophyll cells in mature leaves have fully developed organelles such as chloroplasts, and

47 they usually do not divide in intact plant tissues. When they are isolated by cell wall digestion as

48 single cells called protoplasts and cultivated under phytohormone-containing conditions, however,

49 they reinitiate the cell cycle, grow into an unorganized cell mass called callus and even regenerate

50 whole plants ${ }^{7}$. It is thus clearly demonstrated that differentiated leaf cells can change their fate and

51 take on a meristematic, pluripotent state. Previous studies have described the cytological and

52 physiological properties of protoplasts during cell cycle reinitiation in several plant species,

53 including tobacco (Nicotiana tabacum) and Arabidopsis (Arabidopsis thaliana). Protoplasts, for

54 instance, undergo cell wall reconstruction ${ }^{8,9}$ and changes in the structure and subcellular localization

55 of organelles ${ }^{10,11}$. At the physiological level, cell cycle reinitiation is known to correlate with the

56 neutralization of reactive oxygen species (ROS) ${ }^{12,13}$ and phytohormone production ${ }^{14}$. Additionally,

57 several studies have demonstrated that freshly isolated protoplasts possess a more opened chromatin

58 state compared to intact leaf cells, possibly caused by drastic changes in epigenetic modifications ${ }^{15,16}$.

59 Consistently, Chupeau et al. ${ }^{17}$ reported that Arabidopsis protoplasts undergo dynamic transcriptomic

60 reprogramming during early phases of incubation. Despite these reported findings, the molecular

61 mechanisms that drive cell cycle reinitiation from differentiated cells remain obscure, partly due to a 
62 lack of experimental systems to quantitatively assess this cellular process. To overcome this problem,

63 we improved the culture system for Arabidopsis leaf mesophyll protoplasts and then performed

64 quantitative genetic, physiological and cell biological analyses. Using these approaches, we

65 demonstrate that transcriptional activation of auxin biosynthesis is essential to promote the cell cycle

66 reactivation in differentiated cells.

67

\section{$68 \quad$ Results}

69 Arabidopsis leaf mesophyll protoplasts reprogram and regenerate shoots in vitro.

We first established a new experimental pipeline to reproducibly induce callus formation

71 from Arabidopsis leaf protoplasts (Fig. 1a). Protoplasts were isolated from mature rosette leaves with

72 high viability, embedded into sodium alginate gels and cultured in protoplast callus induction

73 medium (PCIM) supplemented with 2,4-D and thidiazuron as auxin and cytokinin, respectively (Fig.

74 1a, Supplementary Fig. 1a and Supplementary Table 1). After a 14-day incubation, around $2 \%$ of

75 total embedded protoplasts develop into callus, enabling quantitative evaluation of this phenotype

76 (Supplementary Fig.1b). When these protoplast-derived calli are transferred to callus growth medium

77 (CGM) and subsequently to shoot induction medium (SIM), they continue to proliferate and

78 regenerate shoots (Fig. 1b), indicating that these protoplasts acquire competence to form shoot 79 meristems.

80 To uncover the cellular basis for developmental reprogramming, we first determined the

81 original identity of dividing protoplasts. The $p C A B 3: H 2 A-e G F P$ reporter, which drives

82 nuclear-localized accumulation of HISTONE 2A-6 (H2A) fused with enhanced green fluorescent

83 protein (eGFP) under the control of a promoter sequence of a mesophyll cell-specific gene

84 CHLOROPHYLL A/B BINDING PROTEIN $3(C A B 3)^{18}$, is expressed in most freshly isolated

85 protoplasts (Supplementary Fig. 1c). Furthermore, using a time-lapse live imaging system that tracks

86 reprogramming of individual protoplasts over time (Supplementary Fig. 1d), we confirmed that

$8798.78 \%$ (162 out of 164) of protoplasts that undergo cell division display mesophyll cell-like 
88 characteristics at Day 0 based on $\mathrm{H} 2 \mathrm{~A}-\mathrm{eGFP}$ expression and/or the appearance and density of 89 chloroplasts $^{19}$ (Supplementary Fig. 1e-g). The two remaining H2A-eGFP-negative protoplasts also 90 contain chloroplasts but they resemble guard cells based on chloroplast density ${ }^{19}$ (Supplementary Fig.

91 1h). These observations establish that the majority of protoplasts in our experimental setup initially 92 have mesophyll cell identities.

93 Closer examination of early morphological changes via time-lapse imaging revealed that 94 protoplasts undergo their first cell division after Day 4, and consistent with Chupeau et al. ${ }^{17}$, many 95 divide at Day 6 or Day 7 (Fig1c, Supplementary Fig. 1i). As described previously ${ }^{8,9}$, most of them 96 elongate anisotropically for 1 to 3 days before initial cell division (Supplementary Fig. 1j-k). We also 97 observed protoplasts that elongate or expand isotopically without cell division as well as those that 98 shrink or show no apparent changes in size or shape (Supplementary Fig. 2a-b). We have previously 99 shown that vacuolar morphology, visualized by monomeric GFP (mGFP)-tagged VACUOLAR $100 \mathrm{H}^{+}$-PYROPHOSPHATASE 1 (VHP1), changes dynamically as cells transit from proliferative to 101 differentiated phases $^{20}$. Time-lapse imaging of protoplasts carrying $p V H P 1: V H P 1-m G F P$ showed 102 that freshly isolated protoplasts have a single large vacuole occupying most of the cell volume ${ }^{11}$ 103 (Day 0 in Fig. 1c and Supplementary Fig. 2c). Strand-like structures, however, start to appear inside 104 the vacuole either before or when the cells start to elongate and vacuoles undergo extensive 105 compartmentalization as cells progress through successive divisions (Fig. 1c and Supplementary Fig. 106 2c). We observed similar strand-like structures in protoplasts that elongate or expand without cell 107 division but they do not undergo or maintain similar levels of compartmentalization (Supplementary 108 Fig. 2d-e), suggesting that the sustained vacuolar compartmentalization marks protoplasts 109 reprogrammed to divide.

110 In parallel with the reactivation of cell proliferation, cellular dedifferentiation, i.e. the loss of 111 existing traits, is another important aspect of cellular reprogramming. It is previously reported that 112 during in vitro transdifferentiation of mesophyll cells into xylem cells, the expression levels of genes 113 that characterize mesophyll cells, e.g. photosynthetic genes such as CAB3 and RIBULOSE 
114 BISPHOSPHATE CARBOXYLASE SMALL CHAIN $1 A$ (RBCS1A), are immediately decreased,

115 reflecting the loss of mesophyll identity ${ }^{21}$. To investigate when protoplasts start to dedifferentiate, we 116 examined the expression of genes involved in chloroplast functions in mature leaves and protoplasts 117 at early incubation steps by RNA sequencing (Supplementary Fig.3 and Supplementary Table 2). We

118 found that many genes encoding photosynthetic components, including subunits of light harvesting 119 complexes, exhibit striking downregulation during protoplast isolation and/or following several days 120 of incubation. In contrast, genes involved in chloroplast fission, the step important for chloroplast 121 inheritance during cell division, are upregulated within 2 days of incubation, implying that 122 protoplasts initiate transcription to prepare for cell cycle reinitiaton by this time. Importantly, many 123 chloroplast-related genes that become downregulated in cultured protoplasts are known to be 124 significantly upregulated as cells transit from proliferative to differentiated phases in growing 125 Arabidopsis leaves ${ }^{2}$ (Supplementary Fig. 3), implying that the changes in expression of these genes 126 in protoplasts reflects initiation of cellular dedifferentiation. These results thus suggest that cellular 127 dedifferentiation starts as early as during protoplast isolation and the early steps of culture prior to 128 initial cell division.

130 Histone acetylation is required for cell cycle reinitiation in protoplasts.

131 To elucidate the molecular mechanisms of developmental reprogramming, we next focused 132 on the epigenetic modifications that protoplasts undergo during the process. Williams et al. ${ }^{16}$ 133 reported that levels of histone acetylation, which is thought to promote gene expression ${ }^{22}$, increase in 134 freshly isolated protoplasts compared to intact leaves. This suggests that histone acetylation ushers in 135 transcriptional changes in protoplasts during early stages of culture, thus driving developmental 136 reprogramming. Given that GNAT/MYST family histone acetyltransferases (HATs) regulate the 137 expression of key genes in several regeneration contexts, such as during wound-induced callus 138 formation and in vitro shoot regeneration from explants ${ }^{23,24}$, we first tested whether an inhibitor of 139 this family of HATs, MB-3, interferes with protoplast reprograming. As shown in Fig. 2a-b, 
140 application of MB-3 to wild-type (WT) protoplasts strongly reduces callus formation efficiency at 141 Day 14, with protoplasts largely failing to reinitiate cell division. Applying MB-3 at later time points 142 causes similar defects in callus formation, suggesting that histone acetylation is also required for 143 successive cell divisions (Supplementary Fig. 4a). Additionally, an inhibitor of CBP-family HATs, 144 C646, strongly prevents callus formation, whereas garcinol, an inhibitor for p300 and PCAF HATs 145 in human, has a much milder effect (Supplementary Fig. 4b). Furthermore, we found that among 12 146 HATs in Arabidopsis, HISTONE ACETYLTRANSFERASE OF THE GNAT/MYST 147 SUPERFAMILY 1 (HAG1), HAG3 and HISTONE ACETYLATION OF THE TAF $\square 250$ FAMILY 1481 (HAF1) are the key HATs involved in protoplast reprogramming since their single mutants are 149 severely impaired in callus formation (Fig 2a, Supplementary Fig. 4c-e).

150 To investigate how histone acetylation regulates protoplast reprograming, we examined 151 gene expression via RNA-seq in freshly isolated protoplasts as well as in those cultured with or 152 without $50 \mu \mathrm{M}$ MB-3. Considering that the cell division occurs in only a few percent of protoplasts 153 (Supplementary Fig. 2a) and is not synchronized over time (Supplementary Fig. 1i-j), we harvested 154 the protoplasts cultured for 14 days, when we expect that the majority of division-competent 155 protoplasts have undergone the first cell division, to maximize the number of detectable differentially 156 expressed genes. Since histone acetylation is associated with activation of transcription, we focused 157 on the 535 genes that show significantly stronger upregulation in the control condition compared to 158 the MB-3 condition (Fig. 2c and Supplementary Table 3). Gene ontology (GO) analysis of the 535 159 upregulated genes revealed strong fold enrichments of the genes implicated in biosynthesis or 160 metabolism of indole-containing compounds, especially tryptophan-derived ones such as indole 161 glucosinolate and auxin indole-3-acetic acid (IAA) (Supplementary Table 4 and 5). More detailed 162 examination of the expression patterns for a comprehensive set of genes implicated in tryptophan 163 metabolism revealed that some IAA biosynthesis genes, including YUCCAI (YUC1) and 164 CYTOCHROME P450 genes (CYPs), are expressed at higher levels in the control condition 165 compared to the MB-3 condition (Fig. 2d and Supplementary Table 6a). Although statistically not 
significant, we also observed upregulation of more numbers of other YUCs in the control condition,

167 suggesting that IAA biosynthesis is repressed by MB-3 treatment. To test if IAA biosynthesis is 168 required for cell cycle reinitiation, we examined whether blocking synthesis of this hormone impedes 169 callus formation. As shown in Fig. 2e and Supplementary Fig. 5a, callus formation is strongly 170 compromised in WT protoplasts treated with the IAA biosynthesis inhibitors yucasin and Kyn. We

171 also observed similar defects in two mutants defective in IAA biosynthesis, yuc3 yис5 yис7 yuc8 $172 y$ yс $9(y u c Q)^{25}$ and cytochrome p450 family 79 subfamily B polypeptide 2 (cyp79B2) cyp79B3 ${ }^{26}$ (Fig. $1732 \mathrm{f}-\mathrm{g}$ ), supporting the idea that IAA biosynthesis promotes protoplast division. Consistently, 174 overexpression of YUC1 in LexA-VP16-estrogen receptor (XVE)-YUC1 protoplasts or application of 175 low concentrations of IAA reproducibly increases callus formation efficiency (Fig. $2 \mathrm{~h}$ and 176 Supplementary Fig. 5b-c), further substantiating that the level of IAA is a key limiting factor for cell 177 cycle reactivation. Interestingly, it seems that $Y U C$ expression within an appropriate dose range is 178 needed to maximally promote cell cycle reinitiation. A low level of leaky YUCl expression under 179 mock treatment or mild induction of the $Y U C 1$ transgene by $0.1 \mu \mathrm{M} \beta$-estradiol (ED) in $X V E-Y U C 1$ 180 are sufficient to increase callus formation efficiency compared to the WT, while strong 181 overexpression of the YUC1 transgene by 1 or $5 \mu \mathrm{M}$ ED does not have this effect (Fig. $2 \mathrm{~h}$ and 182 Supplementary Fig. 5c). Furthermore, our data suggest that the timing of $Y U C$ expression is also 183 important since induction of YUCl transgene at Day 4 results in greater promotion of callus 184 formation compared to its induction at Day 0 (Fig. 2h and Supplementary Fig. 5c). response.

Our results so far suggest that IAA biosynthesis prior to the initial cell division promotes 189 callus formation from mesophyll protoplasts. Indeed, our RNA-seq data at early time points show 190 that IAA biosynthesis genes are upregulated before initial cell division (Fig. 3a and Supplementary 191 Table 6b). These data support the previous findings that the IAA content is transiently increased just 
192 before initial cell division in alfalfa protoplasts ${ }^{14}$. We therefore hypothesized that IAA biosynthesis 193 specifically promotes initial cell division. Notably, yucasin strongly inhibits callus formation only 194 when it is added by Day 4, i.e. before most cells reinitiate the cell cycle (Fig. 3b), indicating that 195 endogenous IAA production is specifically required for initial cell division. To further investigate the 196 role of IAA biosynthesis during early stages of protoplast reprogramming, we visualized the auxin 197 response by time-lapse imaging of protoplasts carrying DR5rev:GFP, which expresses GFP via the 198 auxin response element-containing $D R 5 \operatorname{promoter}^{27,28}$. In the control condition, we barely observed 199 DR5rev:GFP expression in freshly isolated protoplasts, while many start to express detectable GFP 200 signals from Day 2 to Day 4 (Fig. 3c-d), with nearly 50\% of protoplasts showing DR5rev:GFP 201 expression at least at one time point by Day 6. Importantly, we further found that IAA biosynthesis is 202 required for activation of the auxin response, since yucasin treatment severely reduces the proportion 203 of DR5rev:GFP-positive protoplasts (Fig. 3d). We should also note that DR5rev:GFP-positive cells 204 in the control condition are enriched among protoplasts that divide, elongate or expand, whereas 205 DR5rev:GFP-negative protoplasts in either the control or yucasin condition generally decrease in 206 size (Supplementary Fig. 6a-b). These observations suggest that DR5rev:GFP-detectable auxin 207 response promotes the early phases of developmental reprograming although that alone is not 208 sufficient to complete the first cell division.

210 Auxin biosynthesis is required to transcriptionally activate G2/M phase genes.

211 To further reveal how auxin promotes resumption of the mitotic cell cycle, we compared 212 expression patterns of core cell cycle regulators under control and yucasin conditions by RNA-seq 213 (Fig. 4a and Supplementary Table 7). Previous studies have shown that freshly isolated leaf 214 mesophyll protoplasts reside at the G1 phase and enter the S phase only upon phytohormone 215 application $^{15}$. Consistently, genes functioning during the $\mathrm{G} 1$ to $\mathrm{S}$ phase, including D-type CYCLINs 216 (CYCDs) and MINICHROMOSOME MAINTENANCE 3 (MCM3), show upregulation from Day 2 in 217 control condition. In agreement with our observation that the timing of initial cell division peaks 
218 around Day 6, genes functioning during the G2 to M phase, such as B-type CYCLIN-DEPENDENT 219 KINASEs (CDKBs), B-type CYCLINs (CYCBs), CELL DIVISION CYCLE 20.1 (CDC20.1) and $220 C D C 20.2$, are upregulated particularly at Day 4 and Day 6. Strikingly, in the yucasin condition, the 221 expression of G1/S genes is comparable to that in the control condition, while many G2/M genes are 222 clearly suppressed (Fig. 4a). This notion is further confirmed when a larger set of genes that show 223 transcriptional activation in $\mathrm{S}$ phase or $\mathrm{G} 2 / \mathrm{M}$ phase is examined ${ }^{29}$ (Supplementary Fig. 7a and 224 Supplementary Table $7 \mathrm{a}-\mathrm{c}$ ), suggesting that auxin biosynthesis is required to transcriptionally 225 activate the G2/M phase genes. We also observed similar transcriptional trends in the MB-3 226 condition (Fig. 4a, Supplementary Fig. 7a), supporting our hypothesis that one of the key 227 downstream pathways regulated by histone acetylation is the IAA biosynthesis-dependent G2/M 228 progression. Intriguingly, yucasin or MB-3 treatment has little impact on the transcription of 229 photosynthetic genes (Supplementary Fig. 8 and Supplementary Table 2), suggesting that this aspect 230 of cellular dedifferentiation is regulated by mechanisms independent from histone acetylation and 231 IAA biosynthesis.

232 The identification of a number of $\mathrm{G} 2 / \mathrm{M}$ genes transcriptionally targeted by auxin 233 biosynthesis and histone acetylation suggests that some master regulators of these genes are involved 234 in this regulation. Previous studies have shown that MYB3R4, together with MYB3R1, functions as 235 a transcriptional activator of G2/M genes and their peak in activity at the G2/M phase is regulated at 236 transcriptional and/or post-translational levels ${ }^{29-32}$. Indded, our RNA-seq data show that the 237 expression of MYB3R4 is sharply upregulated from Day 2 to Day 6 in control but not in either the 238 yucasin or MB-3 condition (Fig. 4a), implying that auxin biosynthesis and histone acetylation 239 regulate cell cycle progression by transcriptionally upregulating MYB3R4. To test whether these 240 activator MYB3Rs regulate cell cycle reentry in protoplasts, we isolated protoplasts from myb3r4-1 241 or myb3r1-1 myb3r4-1 mutants and tested their callus formation efficiencies. As shown in Fig. 4b, 242 myb3r1-1 myb3r4-1 mutants, in which overall expression of G2/M genes are strongly repressed ${ }^{30,31}$, 
243 have severely impaired callus formation efficiency (Fig. 4b), indicating that these activator MYB3Rs

244 are required for cell cycle reinitiation in protoplasts.

245 The discovery that auxin biosynthesis is critical for protoplast cell cycle reinitiation is 246 surprising considering that PCIM contains exogenous auxin, 2,4-D, which is indispensable for initial

247 cell division ${ }^{33}$ (data not shown). Previous studies have indicated that exogenous auxin regulates the

$248 \mathrm{G} 1 / \mathrm{S}$ transition at both transcriptional and post-translational levels in protoplasts ${ }^{33,34}$, raising the

249 possibility that exogenous and endogenous auxin regulate different phases of the cell cycle to 250 reinitiate cell division in protoplasts. To test this hypothesis, we compared the transcriptional 251 activation of S phase and G2/M phase genes in control, yucasin and 2,4-D-omitted (- 2,4-D) culture 252 conditions. Our data show that many of genes essential for the $\mathrm{S}$ phase progression, such as $253 C Y C D 2 ; 1, C Y C D 3 ; 1$ and $M C M 3$, are clearly suppressed in the - 2,4-D condition compared to the 254 control and yucasin conditions, whereas G2/M phase genes show similar levels of hypo-activation in 255 both the yucasin and - 2,4-D conditions (Fig. 4c, Supplementary Fig. 7b and Supplementary Table $2567 \mathrm{~d}-\mathrm{e})$. We also found that KIP-RELATED PROTEIN 1 (KRP1), an inhibitor for CDKA/CYCD 257 complexes, is strongly overexpressed in the - 2,4-D condition (Fig. 4c), further supporting 258 transcriptional repression of the G1/S transition. These results therefore suggest that exogenous and 259 endogenous auxin have distinct roles during the initial division of protoplasts, where they 260 transcriptionally activate the S phase and G2/M phase progression, respectively.

ARF7/ARF19 and IAA3/IAA18-mediated auxin signalling pathway regulates cell cycle reinitiation in protoplasts. to investigate which auxin signalling pathways participate in this regulation. As reported previously, 266 auxin is also critical for other forms of cellular reprogramming in plants, including pluripotent callus 267 formation from explants in tissue culture ${ }^{4}$. In Arabidopsis auxin promotes cell cycle reactivation in 268 pericycle cells on callus induction medium (CIM), and this is mediated by ARF7, ARF19, IAA14 
270 many of these auxin signalling regulators are sharply upregulated before initial cell division of 271 protoplasts (Fig. 5a and Supplementary Table 8), implying that they also participate in cell cycle 272 reinitiation from differentiated leaf cells. A loss-of-function mutant for ARF7, non-phototrophic 273 hypocotyl 4-1 (nph4-1 $)^{38}$, indeed displays defects in the auxin response in mesophyll protoplasts ${ }^{39}$

274 and consistently, single or double mutants for ARF7 and ARF19 are impaired in callus formation 275 from protoplasts (Fig. 5b, Supplementary Fig. 9a). Intriguingly, however, a gain-of-function mutant 276 for IAA14, solitary root-1 $(s / r-1)^{40}$, and two loss-of-function mutants for $L B D$ s, 277 pLBD16:LBD16-SRDX and lbd16-1 lbd18-1 lbd33-1 $1^{41}$ make callus from protoplasts with the similar 278 efficiency as the WT, despite displaying severe defects in callus formation from hypocotyl explants 279 in tissue culture ${ }^{35}$ (Fig. 5b-c, Supplementary Fig. 10). These results indicate that reprogramming 280 from differentiated leaf cells is regulated by distinct auxin signalling pathways. To further investigate 281 the auxin signalling components responsible for protoplast reprogramming, we selected other 282 Aux/IAA candidates that have expression levels comparable with those of ARF7 and/or ARF19 in 283 leaves and show strong binding to these $\mathrm{ARFs}^{42}$ (Supplementary Fig. 9b and Supplementary Table 9).

284 Among them, we found that gain-of-function mutants for IAA3 and IAA18, suppressor of hy2-101 $285(\operatorname{shy2}-101)^{43}$ and crane $-2^{44}$, respectively, strongly inhibit callus formation from protoplasts while a 286 gain-of-function mutation in IAA7, auxin resistant $2-1$ (axr2-1 $)^{45}$ does not cause obvious defects (Fig.

287 5d). Collectively, our results suggest that IAA3 and IAA18, together with ARF7 and ARF19, 288 mediate the auxin response in protoplasts, which drives cell cycle reinitiation.

\section{Discussion}

In this study we demonstrate that one of the key factors that permit reprogramming of 292 differentiated plant cells is the activation of auxin biosynthesis. We show that endogenously 293 produced IAA is required to increase auxin response in protoplasts, thereby inducing the expression 294 of G2/M genes to complete cell division (Fig. 6). Notably, auxin biosynthesis is critical specifically 
295 for their first cell division while it is not essential for successive divisions (Fig. 3b). Since 296 differentiated cells should be equipped with mechanisms to prevent ectopic cell proliferation ${ }^{46}$, it is 297 plausible that cell cycle reinitiation in protoplasts requires some unique mechanisms in addition to 298 those functioning during successive cell proliferation, with the latter more similar to those at play in 299 normal development. Previous studies, for instance, have shown that differentiating leaf cells 300 accumulate regulators, such as DA1 and MEDIATOR 25 (MED25), that repress proliferation and 301 promote cellular growth ${ }^{47}$. In parallel, they shut down the expression of many cell cycle activators 302 such as CYCD4s and ANAPHASE COMPLEX 10 (APC10) through epigenetic mechanisms ${ }^{46,48}$. 303 Differentiating cells, additionally, develop physical properties such as thickened cell walls and 304 enlarged vacuoles that may inhibit cell division. Restarting the cell cycle should therefore require the 305 removal of these negative factors and/or induction of some potent activators that can drive cell cycle 306 reentry. In the case of mesophyll protoplasts, auxin biosynthesis induces the transcription of G2/M 307 phase genes likely through MYB3R4 and MYB3R1 (Fig. 4), suggesting that these MYB3Rs serve as 308 key re-activators of cell division. These MYB3Rs appear to be dispensable for mesophyll cell 309 proliferation in intact leaves ${ }^{31}$, so they might be required to induce cell division specifically in 310 differentiated cells where other factors promoting cell cycle progression are possibly inactivated.

311 How auxin biosynthesis regulates MYB3R4/1-mediated pathways is an important question, and its 312 mechanistic details should be further investigated in future studies. Our data suggest that auxin 313 biosynthesis transcriptionally activates MYB3R4 (Fig.4a), but it, as well as MYB3R1, might be also 314 subject to post-translational regulation since phosphorylation and temporal nuclear shuttling of 315 MYB3Rs are central for their functions during mitosis ${ }^{32,49}$.

316 We predict that exogenously supplied 2,4-D serves as another strong driver of cell cycle 317 reinitiation in protoplasts and interestingly, our data suggest that 2,4-D and endogenously produced 318 IAA activate distinct phases of the cell cycle to reinitiate protoplast division (Supplementary Fig. 7b). 319 This is consistent with earlier reports that exogenous 2,4-D is required during the initial steps of 320 protoplast culture ${ }^{33}$, while IAA biosynthesis is activated later on (Fig. 2h; Pasternak et al., 2002). To 
321 further clarify how these two auxins cooperate to promote cell cycle reinitiation, it will be helpful to 322 investigate whether the ARF7/19- and IAA3/18-mediated pathway transduces 2,4-D- and/or 323 IAA-induced signalling. Alternatively, it is possible that stimulation of the IAA biosynthetic pathway 324 itself plays a crucial role in protoplast division. For instance, the YUC cofactor flavin adenine 325 dinucleotide (FAD) is oxidized and produces hydrogen peroxide $\left(\mathrm{H}_{2} \mathrm{O}_{2}\right)$ when the IAA precursor 326 indole 3-pyruvate (IPA) is deficient ${ }^{50} . \mathrm{H}_{2} \mathrm{O}_{2}$ has been demonstrated to either promote or inhibit cell 327 cycle reinitiation in protoplasts depending on the timing of its production and subcellular 328 localization ${ }^{12,13}$. Since $Y U C$ genes also need to be upregulated at an appropriate dose and time during 329 protoplast culture (Fig. 2h), activation of the IAA biosynthetic pathway may contribute to protoplast 330 reprogramming through the production of its byproducts such as $\mathrm{H}_{2} \mathrm{O}_{2}$. It is also worth considering 331 the possibility that overall regulation of indole-containing compound metabolism affects protoplast 332 reprogramming, since our RNA-seq data show the striking upregulation of the indole 333 metabolism-related genes that may not act in the IAA biosynthetic pathway (Fig. 2e; Supplementary

334 Table 4). Investigating the potential contribution of these non-hormonal metabolites may help 335 uncover a new regulatory mechanism underlying plant cellular reprogramming.

\section{Methods}

\section{Plant materials and growth conditions}

339 Arabidopsis thaliana (L) Heynh. ecotype Columbia-0 (Col-0) was used as the wild type. For 340 phenotypic analyses, hag1-1 (SALK_150784), hag1-2 (SALK_030913), hag2 (SALK_051832), 341 hag3-1 (GABI_555H06), hag3-2 (SALKseq_060819), ham1 (SALK_027726), ham2 342 (SALK_106046), hac1 (SALK_080380), hac2 (SALK_049434), hac4 (SALK_045791), hac5 343 (SAIL_49_D10), hac12 (SALK_012469), haf1-1 (SAIL_256_D10), haf1-2 (SALK_110848), haf2 344 (SALK_110029), уисQ (GABI_376G12 x CSHL_GT6160 x SALK_059832 x SM_3_23299 x 345 SALK_762_D07) ${ }^{25}, \quad$ myb3r4-1 (SALK_059819), myb3r1-1 myb3r4-1 (SALK_018482 x 346 SALK_059189) ${ }^{30}$, arf7-1 (SALK_040394), arf19-4 (SALK_009879), nph4-1 arf19-1 ${ }^{38}$, arf7-2 
$\operatorname{arf19-5^{41},}$ slr-1 $1^{40}, l b d 16-1$ lbd18-1 lbd33-1 (SALK_095791 x SALK_038125 x SAIL_95_H10) ${ }^{41}$,

348 axr $2-1^{45}$, shy2-101 $1^{43}$ and crane- $2^{44}$ were used. cyp79B2 cyp79B3 mutants were generated by crossing 349 cyp79B2 (SALK_130570C) with cyp79B3 (SALKseq_066556). The $p V H P 1: V H P 1-m G F P^{21}$ and

350 DR5rev:GFP lines ${ }^{28}$ were previously described. All these mutants and transgenic plants were in

351 Col-0 background. Seeds were sterilized with $70 \%$ ethanol for 1 min and $20 \%$ chlorine bleach

352 (Kao) for $10 \mathrm{~min}$, and then rinsed by autoclaved water for 3 times. After being soaked in water at $4 \square$

353 for 2 to 4 days, seeds were sown on gemination medium (GM) (Supplementary Table 1) with the 354 density of 33 seeds per 90-mm diameter and 25-mm thick polystyrene dish (Kord-Valmark). Plants 355 were grown at $22 \square$, under continuous light (30 to $40 \mu \mathrm{mol} / \mathrm{m}^{2} / \mathrm{s}$ ) in a growth chamber (Sanyo).

357 Transgenic plants

358 To construct the $p C A B 3: H 2 A-e G F P$ vector, $C A B 3$ promoter, 1,537 bp upstream of the ATG start 359 codon, was amplified from genomic DNA by PCR. The PCR products were purified and cloned into 360 the pDONRP4P1r vector (Thermo Fisher Scientific). The promoter fragment was then assembled 361 with GAL4 into the $p B-9 F H 2 A-U A S-7 m 24 G W$ destination vector in a multi-site gateway reaction to 362 create an activator line construct using the LR Clonase II+ (Thermo Fisher Scientific). This 363 destination vector contains a HISTONE 2A-6 (H2A) encoding sequence fused to eGFP and driven 364 by the repetitive UAS promoter, as described by Fendrych et al. (2014) ${ }^{51}$. To construct the pHAG1:HAG1-GFP vector, HAG1 promoter, 1,987 bp upstream of the ATG start codon, was amplified from genomic DNA, purified and cloned into the pDONRP4P1r vector (Thermo Fisher

367 Scientific). HAG1 coding sequence was amplified from complementary DNA (cDNA) by PCR, 368 purified and cloned into the $p E N T R / D-T O P O$ vector (Thermo Fisher Scientific). The promoter and 369 coding sequence fragments were then assembled with GFP into the $R 4 p G W B 504$ destination vector 370 in a multi-site gateway reaction using the Gateway LR Clonase $\square$ (Thermo Fisher Scientific). To 371 construct the $X V E-Y U C 1$ vector, $Y U C 1$ coding sequence was amplified from cDNA by PCR. The 372 PCR products were purified and cloned into $p E N T R / D-T O P O$ vector (Thermo Fisher Scientific). The 
373 cDNA fragments were then cloned into the modified $p E R 8$ plasmid containing a Gateway cassette

$374(p E R 8-G W)^{52}$ using the Gateway LR Clonase $\square$ (Thermo Fisher Scientific). For plant transformation,

375 the plasmids were introduced into Agrobacterium tumefaciens (strain GV3101) by electroporation

376 and transformed into Arabidopsis Col-0 WT plants (for $p C A B 3: H 2 A-e G F P$ and XVE-YUC1) or

377 hag1-1 heterozygous plants (for $p H A G 1: H A G 1-G F P$ ) by the floral dip method ${ }^{53}$. As for 378 pHAG1:HAG1-GFP/hag1-1 plants, after the selection of single insertion lines of 379 pHAG1:HAG1-GFP at T2, the lines homozygous for hag1-1 mutation were further selected by PCR 380 genotyping. Lines homozygous both for $p H A G 1: H A G 1-G F P$ and hagl-1 were then selected at T3. A 381 list of primers used for PCR is described in Supplementary Table 10.

\section{Chemical compounds}

384 2,4-dichlorophenoxyacetic acid (2,4-D) (Cas 94-75-7, Sigma), C646 (Cas 328968-36-1, Sigma) ${ }^{54}$, $385 \beta$-estradiol (ED) (Cas 50-28-2, Wako), garcinol (Cas 78824-30-3, Focus biomolecules) ${ }^{55}$, kinetin 386 (Kin) (Cas 525-79-1, SIGMA), L-kynurenine (Kyn) (Cas 2922-83-0, TCI) ${ }^{56}, \gamma$-butyrolactone (MB-3) 387 (CAS 778649-18-6, Abcam) ${ }^{57}$, thidiazuron (TDZ) (Cas 51707-55-2, Wako), 388 5-(4-chlorophenyl)-4H-1,2,4-triazole-3-thiol (yucasin) (CAS 26028-65-9, Wako) ${ }^{58}$, 389 6-( $\gamma, \gamma$-Dimethylallylamino)purine (2-iP) (CAS 2365-40-4, Sigma), were dissolved in dimethyl 390 sulfoxide (DMSO) and sterilized by filtration. Indole-3-acetic acid (IAA) (Cas 6505-45-9, Wako) 391 was dissolved in ethanol and sterilized by filtration. Fluorescein diacetate (FDA) (Cas 596-09-8, 392 DOJINDO) was dissolved in DMSO and used without sterilization. All chemicals were stored at $393-20 \square$.

\section{Protoplast isolation and callus induction}

396 Protoplasts were isolated following Damm and Willmitzer ${ }^{59}$ with modifications. All processes were 397 performed at room temperature (22 to $25 \square$ ) unless otherwise specified. 1st to 5th rosette leaves of 23 398 or 24 days after sowing (DAS) plants were aseptically harvested, and after carefully removing 
petioles, chopped into strips with a scalpel (Akiyama) in $0.5 \mathrm{M}$ mannitol. After 1-hour maceration in 0.5 M mannitol under dim light, leaf strips from 100 to 150 leaves were transferred into a $35-\mathrm{mm}$ polystyrene petri dish (FALCON) and soaked in $3 \mathrm{~mL}$ of Digestion Cocktail (Supplementary Table 1). For cell wall digestion, leaf strips were gently shaken horizontally on Shake-LR (TAITEC) for 3 nylon Cell Strainer (FALCON) with gentle pipetting using a Komagome type pipette (IWAKI). The filtrate was collected into a $12 \mathrm{~mL}$ culture tube with a conical bottom (Simport), diluted with $1 / 2$ volume of $0.2 \mathrm{M} \mathrm{CaCl}_{2}$ and centrifuged for $5 \mathrm{~min}$ at $60 \mathrm{~g}$ in a swinging bucket rotor (CF16RN, HITACHI), with the slowest acceleration and no brake. The pellet was resuspended in $5 \mathrm{~mL}$ of Wash Medium 1 (Supplementary Table 1) and centrifuged for $3 \mathrm{~min}$ at $40 \mathrm{~g}$. The resulting pellet was resuspended in $5 \mathrm{~mL}$ of Wash Medium 2 (Supplementary Table 1) and centrifuged again. The pellet 410 was subsequently resuspended in $5 \mathrm{~mL}$ of $0.5 \mathrm{M}$ mannitol and centrifuged again. The protoplasts 411 were finally resuspended in fresh $0.5 \mathrm{M}$ mannitol and placed on ice for $40 \mathrm{~min}$ to 1 hour under dim 412 light. To test the viability of fresh protoplasts, FDA was added to an aliquot of protoplast solution at 413 the final concentration of $1 \mathrm{mg} / \mathrm{L}$ and incubated for $10 \mathrm{~min}$. The stained cells were mounted onto a 414 microscope slide and observed with a fluorescence microscope (BX51, OLYMPUS). After 415 calculating cell density with a hemocytometer (Sunlead Glass Corp.), the protoplasts were warmed to room temperature and their density was adjusted to 4.8 to $5.0 \times 10^{5}$ cells $/ \mathrm{mL}$ with $0.5 \mathrm{M}$ mannitol. 
425 (Supplementary Table 1). The plates and dishes were then sealed with surgical tape and parafilm, 426 respectively, and embedded protoplasts were aseptically cultured at $22 \square$ in the dark.

427 Callus formation efficiency was evaluated at Day 14 unless otherwise specified. Calli 428 embedded in each sodium alginate gel were counted under a dissection microscope (M165 FC, 429 Leica) at $2.5 \times$ magnification. Callus formation efficiency was calculated as a percentage of the 430 number of calli in the number of protoplasts initially contained in one sodium alginate gel. The 431 reproducibility was confirmed in at least 3 biological replicates. As the control experiments in 432 chemical treatment assays, protoplasts of corresponding genotypes were treated with the equivalent 433 volumes of solvents (DMSO or ethanol) to confirm that their callus formation efficiencies are not 434 affected by these treatments (data not shown in some figures).

435

436 Induction of shoot formation from protoplast-derived callus

437 After incubating in PCIM for 14 days, calli-containing gels were transferred to $4 \mathrm{~mL}$ of callus 438 growing medium (CGM) (Supplementary Table 1) and cultured at $22 \square$ under continuous dim light 439 (10 to $12 \mu \mathrm{mol} / \mathrm{m}^{2} / \mathrm{s}$ ). CGM was refreshed every 2 weeks. For induction of de novo shoot formation, 440 calli were isolated from the gels by shaking them in Citrate Solution (Supplementary Table 1) for 1 441 to 2 hours, which chelates calcium ions and dissolves the gel. Isolated calli were washed twice by 442 liquid Gamborg B5 medium and cultured on shoot induction medium (SIM) (Supplementary Table $4431)$ at $22 \square$ under continuous light $\left(22-28 \mu \mathrm{mol} / \mathrm{m}^{2} / \mathrm{s}\right)$.

\section{Callus induction from etiolated hypocotyls}

446 To induce callus from tissues, seeds were sown on GM for Tissue Culture (Supplementary Table 1) 447 and grown at $22 \square$ in the dark for 7 days. Hypocotyls of etiolated seedlings were excised into around 448 5-mm-long explants using a scalpel and incubated on Callus Induction Medium (CIM) 449 (Supplementary Table 1 ) for 21 days at $22 \square$ under continuous light $\left(22-28 \mu \mathrm{mol} / \mathrm{m}^{2} / \mathrm{s}\right)$. The 450 reproducibility was confirmed in 2 biological replicates. 


\section{Live imaging of protoplast reprogramming}

453 The microscopic observation method for tracking identical protoplasts was developed based on Hall 454 et $a l .{ }^{62}$ and Dovzhenko et al. ${ }^{63}$. To immobilize the protoplast-containing gels on the bottom of a 455 35-mm petri dish, a polypropylene grid $(1.7 \times 1.7 \mathrm{~mm}$ mesh size, NIP), cut into $11 \times 11$ meshes, were 456 placed a $\mathrm{CaCl}_{2}$ Plate and the protoplast solution was solidified on the mesh. The gel was 457 subsequently fixed to the bottom of a petri dish using the attached grid and additional 4 gels were 458 placed to the same dish to keep the number of protoplasts within a well constant as in our standard 459 culture condition. These gels were cultured with $4 \mathrm{~mL}$ of PCIM at $22 \square$ in the dark.

460 Time-lapse imaging was performed using a confocal microscope (TCS-SP5, Leica) with a 461 water immersion lens (HC FLUOTAR L 25×/ 0.95 W VISIR, Leica). The protoplast-containingpetri 462 dishes were placed on the microscopic stage at each time of observation. Region of Interests were 463 manually tracked based on the coordinates (x, y, z) recorded on the first day. Z-stacked images were 464 taken at $6 \mu \mathrm{m}$ intervals to maximize the number of protoplasts that can be tracked. Each observation 465 was performed within 1 hour per dish to avoid excessive light exposure of protoplasts. Images were 466 processed and analyzed with Fiji ver. 2.0.0 (https://imagej.net/Fiji) and Microsoft Excel. The 467 reproducibility was confirmed in at least 2 biological replicates.

\section{Transcriptome analysis}

470 RNA was extracted from $\sim 1 \mathrm{~g}$ (in fresh weight) of rosette leaves or 1.0 to $1.5 \times 10^{6}$ protoplasts that 471 were prepared from 23 DAS plants. Freshly isolated protoplasts were collected as pellet in a 2-mL 472 tube by centrifugation for $1 \mathrm{~min}$ at 3,200 $\mathrm{g}$ (MX-305, TOMY) and stored at $-80 \square$. To collect cultured 473 protoplasts embedded in the sodium alginate gel, 25 gels per sample were soaked into $25 \mathrm{~mL}$ of

474 Citrate Solution and protoplasts were released by gentle shaking for 1 hour. Three biological 475 replicates were prepared for each time point. Total RNA was isolated using the RNeasy plant mini 476 kit (Qiagen). Isolated RNA was then subjected to library preparation using the Kapa stranded mRNA 
477 sequencing kit (Kapa Biosystems) with NEBNext Multiplex Oligos for Illumina (New England 478 Biolabs) as adapters and Agencourt AMPure XP (Beckman Coulter) beads instead of KAPA Pure 479 Beads. Single-end sequencing was performed on an Illumina NextSeq500 platform. Mapping was 480 carried out using Bowtie ver. 0.12.9. Over $50 \%$ of the reads were uniquely mapped to the TAIR10 481 Arabidopsis genome, resulting in 5 to 17 million mapped reads per sample. Differentially expressed 482 genes were identified using the edgeR package ${ }^{64}$ on R/Bioconductor (https://www.r-project.org/) 483 after normalization of total read counts with Trimmed Mean of M-values (TMM) method.

484 Differentially expressed genes were defined as those that showed $\left|\log _{2} \mathrm{FC}\right|>2$ in transcript levels 485 ( $p$-value < 0.01 and FDR < 0.01). Gene ontology $(\mathrm{GO})$ analyses were performed by PANTHER GO 486 Enrichment Analysis ${ }^{65-67}$ (http://geneontology.org) with PANTHER Overrepresentation Test 487 (Released 20210224). Genes were annotated following GO Ontology database (Released 488 2021-02-01) and categorized by Biological Processes. Venn diagrams were drawn with BioVenn ${ }^{68}$ 489 (http://www.biovenn.nl) and processed with Inkscape ver. 0.92 .4 (https://inkscape.org). Heat maps 490 were drawn using Color Scale tool in Microsoft Excel.

\section{Reverse transcription-quantitative polymerase chain reaction (RT-qPCR)}

$493500 \mathrm{ng}$ of the extracted total RNA were subjected to the first-strand cDNA synthesis with 494 the primescript RT reagent kit (Takara). Quantitative real-time PCR was performed with 495 Thunderbird SYBR qPCR mix (Toyobo) in Stratagene MX3000P real-time qPCR system (Agilent 496 Technologies). The transcript levels were calculated with standard curve method and normalized by 497 that of internal control PROTEIN PHOSPHATASE 2A-3 $(P P 2 A 3)^{69}$. A set of primers used for qPCR 498 is described in Supplementary Table 10.

499

\section{References}


501 1. Beemster, G. T. S. et al. Genome-wide analysis of gene expression profiles associated with cell 502 cycle transitions in growing organs of Arabidopsis. Plant Physiol. 138, 734-743 (2005).

503 2. Andriankaja, M. et al. Exit from proliferation during leaf development in Arabidopsis thaliana: 504 a not-so-gradual process. Dev. Cell 22, 64-78 (2012).

505 3. Dello Ioio, R. et al. A genetic framework for the control of cell division and differentiation in 506 the root meristem. Science 322, 1380-1384 (2008).

507 4. Christianson, M. L. \& Warnick, D. A. Competence and determination in the process of in vitro 508 shoot organogenesis. Dev. Biol. 95, 288-293 (1983).

509 5. Zhang, T.-Q. et al. A two-step model for de novo activation of WUSCHEL during plant shoot $510 \quad$ regeneration. Plant Cell 29, 1073-1087 (2017).

511 6. Ikeuchi, M. et al. Molecular mechanisms of plant regeneration. Annu. Rev. Plant Biol. 70, $512 \quad 377-406(2019)$.

513 7. Takebe, I., Labib, G. \& Melchers, G. Regeneration of whole plants from isolated mesophyll $514 \quad$ protoplasts of tobacco. Naturwissenschaften 58, 318-320 (1971).

515 8. Kao, K. N., Keller, W. A. \& Miller, R. A. Cell division in newly formed cells from protoplasts 516 of soybean. Exp. Cell Res. 62, 338-340 (1970).

517 9. Schilde-Rentschler, L. Role of the cell wall in the ability of tobacco protoplasts to form callus. $518 \quad$ Planta 135, 177-181 (1977).

519 10. Thomas, M. R. \& Rose, R. J. Plastid number and plastid structural changes associated with 520 tobacco mesophyll protoplast culture and plant regeneration. Planta 158, 329-338 (1983).

521 11. Sheahan, M. B., Rose, R. J. \& McCurdy, D. W. Actin-filament-dependent remodeling of the 522 vacuole in cultured mesophyll protoplasts. Protoplasma 230, 141-152 (2007).

523 12. De Marco, A., \& Roubelakis-Angelakis, K., A. The complexity of enzymic control of hydrogen 524 peroxide concentration may affect the regeneration potential of plant protoplasts. Plant Physiol. 525 110, 137-145 (1996). 
13. Tiew, T. W. Y., Sheahan, M. B., \& Rose, R. J. Peroxisomes contribute to reactive oxygen species homeostasis and cell division induction in Arabidopsis protoplasts. Front. Plant Sci. 6, $1-16(2015)$.

14. Pasternak, T. P. et al. The role of auxin, $\mathrm{pH}$, and stress in the activation of embryogenic cell division in leaf protoplast-derived cells of alfalfa. Plant Physiol. 129, 1807-1819 (2002).

15. Zhao, J. et al. Two phases of chromatin decondensation during dedifferentiation of plant cells. J. Biol. Chem. 276, 22772-22778 (2001).

16. Williams, L. et al. Chromatin reorganization accompanying cellular dedifferentiation is associated with modifications of histone $\mathrm{H} 3$, redistribution od HP1, and activation of E2F-target genes. Dev. Dyn. 228, 113-120 (2003).

17. Chupeau, M. C. et al. Characterization of the early events leading to totipotency in an Arabidopsis protoplast liquid culture by temporal transcript profiling. Plant Cell 25, 2444-2463 (2013).

18. Susek, R. E., Ausubel, F. M. \& Chory, J. Signal transduction mutants of Arabidopsis uncouple nuclear $C A B$ and $R B C S$ gene expression from chloroplast development. Cell 74, 787-799 (1993).

19. Pandey, S., Wang, X.-Q., Coursol, S. A. \& Assmann, S. M. Preparation and applications of Arabidopsis thaliana guard cell protoplasts. New Phytol. 153, 517-526 (2002).

20. Segami, S., Makino, S., Miyake, A., Asaoka, M. \& Maeshima, M. Dynamics of vacuoles and $\mathrm{H}^{+}$-pyrophosphatase visualized by monomeric green fluorescent protein in Arabidopsis:

548 22. Shahbazian, M. D. \& Grunstein, M. Functions of site-specific histone acetylation and 549 deacetylation. Annu. Rev. Biochem.76, 75-100 (2007).

550 23. Rymen, B. et al. Histone acetylation orchestrates wound-induced transcriptional activation and 551 cellular reprogramming in Arabidopsis. Commun. Biol. 2, 1-15. 
24. Kim, J.-Y. et al. Epigenetic reprogramming by histone acetyltransferase HAG1/AtGCN5 is 553 required for pluripotency acquisition in Arabidopsis. EMBO J. e98726 (2018).

554 25. Chen, Q. et al. Auxin overproduction in shoots cannot rescue auxin deficiencies in Arabidopsis $555 \quad$ roots. Plant Cell Physiol. 55, 1072-1079 (2014).

556 26. Zhao, Y. et al. Trp-dependent auxin biosynthesis in Arabidopsis: involvement of cytochrome 557 P40s CYP79B2 and CYP79B3. Gen. Dev. 16, 3100-3112 (2002).

558 27. Ulmasov, T., Murfett, J., Hagen, G. \& Guilfoyle, T. J. Aux/IAA proteins repress expression of 559 reporter genes containing natural and highly active synthetic auxin response elements. Plant Cell $560 \quad 9,1963-1971(1997)$.

561 28. Benková, E. et al. Local, efflux-dependent auxin gradients as a common module for plant organ 562 formation. Cell 115, 591-602 (2003).

563 29. Kobayashi, K. et al. Transcriptional repression by MYB3R proteins regulates plant organ growth. 564 EMBO J. 34, 1992-2007 (2015).

565 30. Haga, N. et al. R1R2R3-Myb proteins positively regulate cytokinesis through activation of 566 KNOLLE transcription in Arabidopsis thaliana. Development 134, 1101-1110 (2007).

567 31. Haga, N. et al. Mutations in $M Y B 3 R 1$ and $M Y B 3 R 4$ cause pleiotropic developmental defects and 568 preferential down-regulation of multiple G2/M-specific genes in Arabidopsis. Plant Physiol. 157, $569 \quad$ 706-717 (2011).

570 32. Yang, W. et al. Molecular mechanism of cytokinin-activated cell division in Arabidopsis. $571 \quad$ Science 10.1126/science.abe2305 (2021).

572 33. Carle, S. A., Bates, G. W. \& Shannon, T. A. Hormonal control of gene expression during 573 reactivation of the cell cycle in tobacco mesophyll protoplasts. J. Plant Growth Regul. 17, $574 \quad 221-230(1998)$.

575 34. Pasternak, T. et al. Exogenous auxin and cytokinin dependent activation of CDKs and cell 576 division in leaf protoplast-derived cell of alfalfa. Plant Growth Regul. 32, 129-141 (2000). 
35. Iwase, A. et al. The AP2/ERF transcription factor WIND1 control cell dedifferentiation in 578 Arabidopsis. Curr. Biol. 21, 508-514 (2011).

579

580

581

582

583

584

585

586

587

588

589

590

591

592

593

594

595

596

597

598

599

600

36. Fan, M., Xu, C., Xu, K. \& Hu, Y. LATERAL ORGAN BOUNDERIES DOMAIN transcription factors direct callus formation in Arabidopsis regeneration Cell Res. 22, 1169-1180 (2012).

37. Lee, K., Park, O.-S. \& Seo, P.-J. Arabidopsis ATXR2 deposits H3K36me3 at the promoters of LBD genes to facilitate cellular dedifferentiation. Sci. Signal. 10, eaan0316 (2017).

38. Okushima, Y. et al. Functional genomic analysis of the AUXIN RESPONSE FACTOR gene family members in Arabidopsis thaliana: unique and overlapping functions of ARF7 and ARF19. Plant Cell 17, 444-463 (2005).

39. Wang, S., Tiwari, S. B., Hagen, G. \& Guilfoyle, T. J. AUXIN RESPONSE FACTOR7 restores the expression of auxin-responsive genes in mutant Arabidopsis leaf mesophyll protoplasts. Plant Cell 17, 1979-1993 (2005).

40. Fukaki, H., Tameda, S., Masuda, H. \& Tasaka, M. Lateral root formation is blocked by $\mathrm{g}$ gain-of-function mutation in the SOLITARY-ROOT/IAAI4 gene of Arabidopsis. Plant J. 29, 153-168. (2002).

41. Goh, T., Joi, S., Mimura, T. \& Fukaki, H. The establishment of asymmetry in Arabidopsis lateral root founder cells is regulated by LBD16/ASL18 and related LBD/ASL proteins. Development 139, 883-893 (2012).

42. Piya, S., Shrestha, S. K., Binder, B., Stewart Jr., C. N. \& Hewezi, T. Protein-protein interaction and gene co-expression maps of ARFs and Aux/IAAs in Arabidopsis. Front. Plant Sci. 5, 1-9 (2014).

43. Goh, T., Kasahara, H., Mimura, T., Kamiya, Y. \& Fukaki, H. Multiple AUX/IAA-ARF modules regulate lateral root formation: the role of Arabidopsis SHY2/IAA3-mediated auxin signalling. Phil. Trans. R. Soc. B 367, 1461-1468 (2012). 
44. Uehara, T., Okushima, Y., Mimura, T., Tasaka, M. \& Fukaki, H. Domain II mutations in CRANE/IAA18 suppress lateral root formation and affect shoot development in Arabidopsis thaliana. Plant Cell Physiol. 49, 1025-1038 (2008).

604

45. Nagpal, P. et al. AXR2 encodes a member of the Aux/IAA protein family. Plant Physiol. 123,

605 $563-573(2000)$.

606

46. Ikeuchi, M., Iwase, A. \& Sugimoto, K. Control of plant cell differentiation by histone modification and DNA methylation. Curr. Opin. Plant Biol. 28, 60-67 (2015).

47. Kalve, S., De Vos, D. \& Beemster, G. T. S. Leaf development: a cellular perspective. Front. Plant Sci. 5, 1-25 (2014).

48. Candeale, J. et al. Differential methylation during maize leaf growth targets developmentally 611 regulated genes. Plant Physiol. 164, 1350-1364 (2014).

612 49. Chen, P. et al. Arabidopsis R1R2R3-Myb proteins are essential for inhibiting cell division in 613 response to DNA damage. Nat. Commun. 8, 1-12 (2017).

614 50. Dai, X. et al. The biochemical mechanism of auxin biosynthesis by an Arabidopsis YUCCA 615 flavin-containing monooxygenase. J. Biol. Chem. 288, 1448-1457 (2013).

616 51. Fendrych, M. et al. Programmed cell death controlled by ANAC033/SOMBRERO determines 617 root cap organ size in Arabidopsis. Curr. Biol. 24, 931-940 (2014).

618 52. Favero, D. S. et al. AT-hook transcription factors restrict petiole growth by antagonizing PIFs. 619 Curr. Biol. 30, 1454-1466 (2020).

620 53. Clough, S. J. \& Bent, A. F. Floral dip: a simplified method for Agrobacterium-mediated 621 transformation of Arabidopsis thaliana. Plant J. 16, 735-743 (1998).

622 54. Bowers, E. M. et al. Virtual ligand screening of the p300/CBP histone acetyltransferase: 623 identification of a selective small molecule inhibitor. Chem. Biol. 17, 471-482 (2010).

624 55. Balasubramanyam, K. et al. Polyisoprenylated benzophenone, garcinol, a natural histone 625 acetyltransferase inhibitor, represses chromatin transcription and alters global gene expression. $J$. 626 Biol. Chem. 279, 33716-33726 (2004). 
56. He, W. et al. A small-molecule screen identifies L-kynurenine as a competitive inhibitor of

628 TAA1/TAR activity in ethylene-directed auxin biosynthesis and root growth in Arabidopsis. Plant Cell 23, 3944-3960 (2011).

57. Biel, M., Kretsovali, A., Karatzali, E., Papamatheakis, J. \& Giannis, A. Design, synthesis, and biological evaluation of a small-molecule inhibitor of the histone acetyltransferase Gcn5. Angew. Chemie. - Int. Ed. 43, 3947-3976 (2004).

633 58. Nishimura, T. et al. Yucasin is a potent inhibitor of YUCCA, a key enzyme in auxin 634 biosynthesis. Plant J. 77, 352-366 (2014).

635 59. Damm, B. \& Willmitzer, L. Regeneration of fertile plants form protoplasts of different 636 Arabidopsis thaliana genotypes. Mol. Gen. Genet. 213, 15-20 (1988).

637 60. Masson, J. \& Paszkowski, J. The culture response of Arabidopsis thaliana protoplasts is 638 determined by the growth conditions of donor plants. Plant J. 2, 829-833 (1992).

639 61. Chupeau, M. C., Lemoine, M. \& Chupeau, Y. Requirement of thidiazuron for healthy protoplast 640 development to efficient tree regeneration of a hybrid poplar (Populus tremula $\beta$ p. alba). J. $641 \quad$ Plant Physiol. 141, 601-609 (1993).

642 62. Hall, R. D., Verhoeven, H. A. \& Krens, F. A. Computer-assisted identification of protoplasts 643 responsible for rare division events reveals guard-cell totipotency. Plant Physiol. 107, $644 \quad 1379-1386(1995)$.

645 63. Dovzhenko, A., Bergen, U. \& Koop, H.-U. Thin-alginate-layer technique for protoplast culture 646 of tobacco leaf protoplasts: shoot formation in less than two weeks. Protoplasma 204, 114-118 (1998).

648 64. Robinson, M. D., McCarthy, D. J. \& Smyth, G. K. edgeR: a Bioconductor package for 649 differential expression analysis of digital gene expression data. Bioinformatics 26, 139-140 $650 \quad(2010)$.

651 65. Ashburner, M. et al. Gene ontology: tool for the unification of biology. Nat. Gen. 25, 25-29 $652 \quad(2000)$. 
66. Carbon, S. et al. The gene ontology resource: 20 years and still GOing strong. Nucleic Acid Res. 47, D330-D338 (2019).

67. Mi, H., Muruganujan, A., Ebert, D., Huang, X. \& Thomas, P. D. PAHTER version 14: more genomes, a new PANTHER GO-slim and improvements in enrichment analysis tools. Nucleic Acid Res. 47, D419-D426 (2019).

68. Hulsen, T., De Vlieg, J. \& Alkema, W. BioVenn - a web application for the comparison and visualization of biological lists using area-proportional Venn diagrams. BMC Genomics 9, 1-6 (2008).

69. Rymen, B. et al. ABA suppresses root hair growth via the OBP4 transcriptional regulator. Plant Physiol. 173, 1750-1762 (2017).

70. Sugawara, S. et al. Distinct Characteristics of indole-3-acetic acid and phenylacetic acid, two

\section{Acknowledgements}

667 We thank Munetaka Sugiyama (the University of Tokyo) for technical advice on protoplast culture, 668 Masaki Ito (Kanazawa University) for the seeds of MYB mutants and Hidehiro Fukaki (Kobe 669 University) for the seeds of ARF and Aux/IAA mutants. This work was supported by a grant from 670 the Ministry of Education, Culture, Sports, and Technology of Japan to K.S. (20H03284 and 671 20H05911) and Grant-in-Aid for JSPS Fellows to Y.S. (20J20380). Y.S. is supported by JSPS DC 672 research fellowship.

673

\section{Author contributions}

675 Y.S. and K.S. conceived the project. Y.S. and K.S. designed the experiments, and Y.S. conducted 676 most of genetic and cell biological analyses except for RNA sequencing and mapping which were 677 performed by T.S. A.K. generated pHAG1:HAG1-GFP/hag1-1 plants. S.P. and L.D.V. generated 
pCAB3:H2A-eGFP plants. S.S. and M.M. provided $p V H P 1$ :VHP1-mGFP seeds. Y.S. and K.S. wrote

679 the manuscript with help from the co-authors.

680

\section{Competing interests}

682 The authors declare no competing interests.

683

\section{Additional information}

685 Supplementary data are available for this paper online. Correspondence and requests for materials 686 should be addressed to K.S.

688 Figure legends

689 Figure 1 Leaf mesophyll protoplasts reprogram into a pluripotent state and regenerate 690 shoots in vitro. a, Diagram showing the key steps for protoplast isolation and callus induction. The 691 circles with dashed lines indicate a sodium alginate gel that contains protoplasts. b, Light microscopy 692 images of a freshly isolated leaf mesophyll protoplast (1), a protoplast that has undergone the first 693 cell division (2), callus formed from a protoplast (3 to 5), and new shoots formed from 694 protoplast-derived callus $(\mathbf{6}, 7)$. c, Time-lapse confocal microscopy images of a leaf mesophyll 695 protoplast undergoing cellular reprogramming from Day 0 to Day 10. Vacuolar morphology is 696 visualized by VHP1-mGFP. The double-headed arrow indicates the direction of cell elongation and 697 arrowheads mark the plane of initial cell division. The white arrow highlights the initial appearance 698 of vacuolar strand-like structures. Scale bars are $10 \mu \mathrm{m}(\mathbf{1}$ to $\mathbf{3}$ in $\mathbf{a}), 100 \mu \mathrm{m}$ ( $\mathbf{4}$ to $\mathbf{6}$ in $\mathbf{a}), 1 \mathrm{~mm}(\mathbf{b})$ 699 and $30 \mu \mathrm{m}(\mathbf{c})$.

700

701 Figure 2 Histone acetylation is required to reinitiate cell division in leaf mesophyll 702 protoplasts. a, Callus formation efficiency of MB3-treated WT, hag1-1, hag2 and hag3-1 703 protoplasts at Day 14. MB-3 was added at the beginning of culture (Day 0). Error bars represent 
704 standard error. $\mathrm{n}=40$ from 8 biological replicates for the WT control, 15 from 3 biological replicates 705 for hagl-1 and hag2, and 20 from 4 biological replicates for all others. $* * * P<0.001$ (two-tailed 706 Welch's $t$-test compared to WT control). n.d. not determined. b, Bright field images of WT 707 protoplasts incubated in control (left panel) and $50 \mu \mathrm{M}$ MB-3 condition (right panel) for 14 days.

708 The white arrowhead indicates a single callus. The bottom right insets show confocal microscopy 709 images of callus cells (left panel) and an undivided protoplast (right panel). c, A Venn diagram of 710 genes significantly upregulated in control and $50 \mu \mathrm{M}$ MB-3 conditions. The green circle indicates 711 genes that show significantly higher expression in the control compared to the MB-3 condition at 712 Day 14. Numbers in brackets show total numbers of genes included in each group. The red outline 713 highlights 535 genes that show significantly stronger upregulation in the control compared to the

714 MB-3 condition. d, Heat map of the transcriptional changes for representative genes implicated in 715 IAA biosynthesis. The left two columns show the expression levels in the control and MB-3 716 conditions at Day 14 as values normalized $\left(\log _{2} \mathrm{FC}\right)$ to Day 0 . The 'Relative levels to control' 717 column shows the differential expression levels in the MB-3 condition compared to the control at 718 Day 14 as relative values $\left(\log _{2} \mathrm{FC}\right)$. Average reads per million (RPM) indicates the overall expression 719 level for each gene. Asterisks mark the genes that are included from the 535 upregulated genes in c. e, 720 Callus formation efficiency of yucasin-treated WT protoplasts. Error bars represent standard error. $\mathrm{n}$ $721=30$ from 6 biological replicates for the WT control and 15 from 3 biological replicates for the 722 others. $* * * P<0.001$ (two-tailed Student's $t$-test or Welch's $t$-test compared to WT control). n.d. not 723 determined. f, Callus formation efficiency of WT and yuc3 yuc5 yuc7 yuc8 yuc9 (yucQ) protoplasts.

724 Error bars represent standard error. $\mathrm{n}=15$ from 3 biological replicates. $* * * P<0.001$ (two-tailed 725 Student's $t$-test compared to WT). g, Callus formation efficiency of WT and cyp79B2 cyp79B3 726 protoplasts. Error bars represent standard error. $\mathrm{n}=15$ from 3 biological replicates. $* * * P<0.001$

727 (two-tailed Student's $t$-test compared to WT). h, Callus formation efficiency of XVE-YUC1 728 protoplasts. DMSO or $\beta$-estradiol (ED) were added to PCIM at the indicated final concentration at 729 the beginning (Day 0) or Day 4 of culture. Error bars represent standard error. $\mathrm{n}=15$ from 3 
biological replicates. For 'No treatment', $* * * P<0.001$ (two-tailed Welch's $t$-test compared to WT

731 under the same treatment). For the others, different letters indicate significant differences based on

732 one-way ANOVA with post hoc Tukey's HSD test $(P<0.001)$ within each treatment group. Scale 733 bars are $1 \mathrm{~mm}(\mathbf{b})$ and $50 \mu \mathrm{m}$ (insets in b).

735 Figure 3 Early auxin biosynthesis is required to reinitiate cell division in leaf mesophyll

736 protoplasts. a, Heat map of the transcriptional changes for representative IAA biosynthesis genes at

737 Day 2, 4 and 6 in the control condition. Values $\left(\log _{2} \mathrm{FC}\right)$ are relative to Day 0. Average RPM

738 indicates the overall expression level for each gene. b, Callus formation efficiency of WT protoplasts

739 treated with $50 \mu \mathrm{M}$ yucasin at different time points. Diagram shows the timing of yucasin treatment.

740 Error bars represent standard error. $\mathrm{n}=15$ from 3 biological replicates. $* P<0.05, * * * P<0.001$

741 (two-tailed Welch's $t$-test compared to yucasin treatment at Day 0). c, Time-lapse confocal

742 microscopy images of a mesophyll protoplast that undergoes cell division at Day 5. Auxin response

743 is visualized by DR5rev:GFP expression. d, Frequency of DR5rev:GFP-expressing (positive)

744 protoplasts in the control or $50 \mu \mathrm{M}$ yucasin condition among all tested DR5rev:GFP protoplasts.

745 Error bars represent standard error. $\mathrm{n}=9$ for control and 7 for yucasin-treated protoplasts from 2

746 biological replicates. ${ }^{* * *} P<0.001$ (two-tailed Student's $t$-test or Welch's $t$-test). Scale bars are 30 $747 \mu \mathrm{m}(\mathbf{c})$.

749 Figure 4 | Auxin biosynthesis is required to transcriptionally activate the G2/M phase genes

750 through MYB3R1 and MYB3R4. a, Heat map representing the transcriptional changes for genes

751 encoding core cell cycle regulators at G1/S, S and G2/M phases at Day 2, 4 and 6. The left three

752 columns show expression levels in the control, $50 \mu \mathrm{M}$ yucasin and $50 \mu \mathrm{M}$ MB-3 conditions as values

753 normalized $\left(\log _{2} \mathrm{FC}\right)$ to Day 0. The 'Relative levels to control' columns show the normalized

754 expression levels in the yucasin or MB-3 condition compared to the control condition for respective

755 time points as relative values $\left(\log _{2} \mathrm{FC}\right)$. Average RPM indicates the overall expression level for each 
gene. Gene sets are selected based on Kalve et al. ${ }^{47}$. b, Callus formation efficiency of WT, myb3r4-1

757 and myb3r1-1 myb3r4-1 protoplasts. Error bars represent standard error. $\mathrm{n}=20$ from 4 biological

758 replicates for the WT and myb3rl-1 myb3r4-1 and $\mathrm{n}=15$ from 3 biological replicates for myb3r4-1.

$759 * * * P<0.001$ (two-tailed Student's $t$-test compared to WT). c, Examples of the G1/S phase-related

760 genes that are differently expressed in the - 2,4-D condition compared to the control and yucasin

761 conditions at Day 4. Error bars represent standard error. $n=6$ for Day 0 and the control condition at

762 Day 4 and $\mathrm{n}=3$ for the yucasin and -2,4-D conditions at Day 4

763

764 Figure 5 | ARF7/ARF19 and IAA3/IAA18-mediated auxin signalling pathway drives cell cycle

765 reinitiation in leaf mesophyll protoplasts. a, Heat map representing the transcriptional changes for

766 genes implicated in auxin-induced callus formation in tissue culture. Expression levels at Day 2, 4

767 and 6 in the control condition are shown as values normalized $\left(\log _{2} \mathrm{FC}\right)$ to Day 0 . Average RPM

768 indicates the overall expression level for each gene. Gene sets are taken from Ikeuchi et al. ${ }^{6} . \mathbf{b}$,

769 Callus formation efficiency of WT, nph4-1 arf19-1 and slr-1 protoplasts. Error bars represent

770 standard error. $\mathrm{n}=25$ from 5 biological replicates for the WT and 20 from 4 biological replicates for

771 the others. ${ }^{* * *} P<0.001$ (two-tailed Student's $t$-test or Welch's $t$-test compared to WT). $\mathbf{c}$, Callus

772 formation efficiency of WT, lbd16-1 lbd18-1 lbd33-1 and $p L B D 16: L B D 16-S R D X$ protoplasts. Error

773 bars represent standard error. $\mathrm{n}=20$ from 4 biological replicates for the WT and 15 from 3 biological

774 replicates for the others. No statistical difference was detected (two-tailed Student's $t$-test or Welch's

$775 t$-test compared to WT). d, Callus formation efficiency of WT, shy2-101, axr2-1 and crane-2

776 protoplasts. Since heterozygous and homozygous crane-2 are morphologically indistinguishable,

777 both genotypes were mixed for protoplast preparation. Error bars represent standard error. $n=25$

778 from 5 biological replicates for the WT and 15 from 3 biological replicates for the others. $* P<0.05$,

$779 * * * P<0.001$ (two-tailed Student's $t$-test or Welch's $t$-test compared to WT).

780 
781 Figure 6 Hypothetical model describing the molecular mechanism of developmental

782 reprogramming in leaf mesophyll protoplasts. Freshly isolated Arabidopsis leaf mesophyll

783 protoplasts undergo dramatic developmental reprogramming and produce pluripotent callus when

784 cultured in the presence of auxin and cytokinin. Histone acetylation is required to reinitiate the

785 mitotic cell cycle and one of the key downstream pathways regulated by histone acetylation is the

786 transcription of auxin biosynthesis genes. Endogenously produced auxin, IAA, in turn activates

787 auxin response in protoplasts, possibly in an ARF7/ARF19 and IAA3/IAA18-dependent manner.

788 Endogenous auxin is responsible for the transcriptional upregulation of G2/M genes to complete the

789 initial cell division, and such cell cycle regulation likely involves auxin-dependent induction of

790 MYB3R4. MYB3R4 and MYB3R1 might be also regulated at the post-translational level. Another

791 key feature of developmental reprogramming that protoplasts undergo at an early stage of culture is

792 the cellular dedifferentiation, which is marked, for instance, by drastic downregulation of

793 photosynthetic genes and upregulation of chloroplast fission genes. These transcriptional changes are

794 independent of histone acetylation and auxin biosynthesis. In addition, a large, single vacuole,

795 typical of differentiated cells, becomes compartmentalized and starts to resemble what is found in

796 proliferating or elongating cells.

798 Supplementary Figure legends

799 Supplementary Figure 1 | Cell cycle reinitiation and callus formation described with a newly

800 established culture system and time-lapse confocal microscopy. a, Bright-field and fluorescence

801 microscopy images of freshly isolated WT protoplasts. FDA staining indicates that $98.66 \pm 0.19 \%$

802 (standard error, $\mathrm{n}=10$ ) of freshly isolated protoplasts are viable. $\mathbf{b}$, Callus formation efficiency of

803 WT protoplasts. Average efficiency is $1.98 \pm 0.33 \% . \mathrm{n}=240$ from 48 biological replicates. Each dot

804 shows callus formation efficiency calculated from one gel. In the box plot, the median is represented

805 by a black line and the upper and lower quartiles are represented by the upper and lower ends of the

806 box respectively. c, Bright-field and fluorescence microscopy images of protoplasts freshly isolated 
from plants carrying a mesophyll cell-specific marker $p C A B 3: H 2 A-e G F P$. Quantitative analysis

808 indicates that $94.63 \pm 0.51 \%$ of freshly isolated protoplasts show distinct nuclear-localized

809 H2A-eGFP expression (standard error, $\mathrm{n}=15$ from 2 biological replicates). d, Diagram showing the 810 time-lapse confocal microscopy procedure used to track individual protoplasts. e, Classification of 811164 protoplasts carrying $p C A B 3: H 2 A-e G F P$ that underwent cell division by Day 10 based on 812 H2A-eGFP expression and appearance at Day 0. f, Two examples of $p C A B 3: H 2 A-e G F P$ protoplasts 813 that are H2A-eGFP-positive and mesophyll cell-like at Day 0. Both protoplasts divided by Day 10. g, 814 A $p C A B 3: H 2 A-e G F P$ protoplast that is H2A-eGFP-negative and mesophyll cell-like at Day 0. The 815 protoplast divided by Day 10. h, A $p C A B 3: H 2 A-e G F P$ protoplast that is H2A-eGFP-negative and 816 guard cell-like at Day 0. The protoplasts divided by Day 10. i, Heat maps representing cell size 817 dynamics of protoplasts that reinitiate cell division between Day 4 and Day 9. Each row shows cell 818 diameter (left panel) and change in cell diameter comparted to Day 0 (right panel) for individual 819 protoplasts isolated from WT, DR5rev:GFP or $p V H P 1: V H P 1-m G F P$ plants. Among 94 protoplasts 820 tested in this experiment, 80 cells underwent cell elongation before cell division. $\mathbf{j}$, Timing of cell 821 elongation and cell division for 80 cells from i. k, Duration of cell elongation (right panel) for 80 822 cells from i. Scale bars are $100 \mu \mathrm{m}(\mathbf{a}), 100 \mu \mathrm{m}(\mathbf{d})$ and $20 \mu \mathrm{m}$ (f to h).

823

824 Supplementary Figure 2 Time-lapse confocal microscopy images of leaf mesophyll 825 protoplasts that did or did not undergo cell division. a, Pie chart showing the percentages of 826 protoplasts that underwent cell division (divided), those that elongated without cell division 827 (elongated), those that expanded without cell division (expanded) and those that shrunk or displayed 828 no shape changes (others) among 902 protoplasts isolated form DR5rev:GFP plants and used for 829 time-lapse confocal microscopy. b, Representative time-lapse images of elongated, expanded and 830 other protoplasts from Day 0 to Day 7. c, Another set of time-lapse images of a protoplast that 831 underwent cell division. d, Time-lapse images of a protoplast that elongated without cell division. e, 832 Time-lapse images of a protoplast that expanded without cell division. In $\mathbf{c}$ to e, vacuolar 
833 morphology is visualized by VHP1-mGFP. The double-headed arrow indicates the direction of cell

834 elongation and arrowheads mark the plane of initial cell division. The white arrow highlights the 835 initial appearance of vacuolar strand-like structures. Scale bars are $30 \mu \mathrm{m}$.

837 Supplementary Figure 3 Transcriptional changes of chloroplast-related genes during 838 protoplast reprogramming. Heat map representing the transcriptional changes for genes implicated 839 in chloroplast biogenesis and function. The leftmost column shows relative expression levels 840 ( $\left.\log _{2} \mathrm{FC}\right)$ in protoplasts at Day 0 compared to 23 DAS leaves. The second column shows relative 841 expression levels $\left(\log _{2} \mathrm{FC}\right)$ in cultured protoplasts at Day 2, 4, 6 and 14 compared to Day 0. Average 842 reads per million (RPM) indicates the overall expression level for each gene. The right column 843 shows genes significantly upregulated (orange) or downregulated (blue) during leaf development 844 based on Andriankaja et al. (2012). Gene sets were selected based on annotations at The Arabidopsis 845 Information Resource (TAIR) (https://www.arabidopsis.org/index.jsp).

847 Supplementary Figure 4 Roles of histone acetyltransferases in protoplast cell cycle 848 reinitiation. a, Callus formation efficiency of WT protoplasts treated with $50 \mu \mathrm{M}$ MB-3 at different 849 time points. Diagram shows the timing of MB-3 treatment. Error bars represent standard error. $\mathrm{n}=$ 85015 from 3 biological replicates. n.s. not significant (two-tailed Student's $t$-test or Welch's $t$-test 851 compared to MB-3 treatment at Day 0). b, Callus formation efficiency of WT protoplasts treated 852 with C646 or garcinol. Error bars represent standard error. $n=30$ from 6 biological replicates for the 853 WT control and $\mathrm{n}=15$ from 3 biological replicates for all others. $* * * P<0.001$ (two-tailed Student's $854 t$-test or Welch's $t$-test compared to WT control). n.d. not determined. c, Callus formation efficiency 855 of protoplasts isolated from WT and HAT mutants. Error bars represent standard error. $\mathrm{n}=45$ from 9 856 biological replicates for the WT and $\mathrm{n}=10$ or 15 from 2 or 3 biological replicates for mutants. $* P<$ $8570.05, * * * P<0.001$ (two-tailed Student's $t$-test or Welch's $t$-test compared to WT). d, Callus 858 formation efficiency of WT, hagl-2, hag3-2 and hafl-2 protoplasts. Error bars represent standard 
error. $n=25$ from 5 biological replicates for the WT and $n=15$ from 3 biological replicates for all

860 others. $* * * P<0.001$ (two-tailed Welch's $t$-test compared to WT). e, Callus formation efficiency of

861 WT and pHAG1:HAG1-GFP/hag 1-1 protoplasts. Error bars represent standard error. $\mathrm{n}=15$ from 3

862 biological replicates. No statistical difference was detected (two-tailed Student's $t$-test compared to 863 WT).

865 Supplementary Figure 5| Roles of auxin biosynthesis in protoplast cell cycle reinitiation. a, 866 Callus formation efficiency of Kyn-treated WT protoplasts. Error bars represent standard error. $\mathrm{n}=$ 86730 from 6 biological replicates for the WT control and $n=15$ to 25 from 3 to 5 biological replicates. $868 * * * P<0.001$ (two-tailed Student's $t$-test or Welch's $t$-test compared to WT control). b, Callus 869 formation efficiency of WT protoplasts incubated with PCIM supplemented with 0 to $1,000 \mu \mathrm{g} / \mathrm{L}$ 870 IAA. Error bars represent standard error. $\mathrm{n}=10$ to15 from 2 to 3 biological replicates. $* * P<0.01$, $871 * * * P<0.001$ (two-tailed Student's $t$-test or Welch's $t$-test compared to WT control). c, $872 \beta$-estradiol-inducible expression of YUCl in XVE-YUCl plants. WT and XVE-YUCl seeds were 873 sown in liquid half-strength MS medium and incubated at $22 \square$ under light with rotation on a 874 Shake-LR (TAITEC) for 10 days. The plants were then treated with different concentrations of ED 875 or the equivalent volumes of DMSO and grown for another 24 hours. The expression levels of YUC1 876 are normalized by those of the internal control $P P 2 A 3$ and shown as relative values compared to WT 877 given the same treatments. The $Y U C 1$ expression in WT remained constant throughout the treatments. 878 The numbers above each bar are average expression levels. Error bars represent standard error $(\mathrm{n}=$ $879 \quad 6)$.

881 Supplementary Figure 6 | Correlation between auxin response and the morphological changes 882 during reprogramming of protoplasts. a, Frequency of the DR5rev:GFP-expressing (positive) 883 protoplasts among 902 total cells, 45 divided cells, 221 elongated cells, 89 expanded cells and 547 884 other cells. DR5rev:GFP protoplasts were incubated in the control condition. $* P<0.05$ (two-tailed 
885 Student's $t$-test or Welch's $t$-test compared to 'Total' at the same time point). b, Heat maps 886 representing cell size dynamics of 417 DR5rev:GFP-positive and 485 DR5rev:GFP-negative 887 protoplasts in the control condition. c, Heat maps representing cell size dynamics of 124 888 DR5rev:GFP-positive and 606 DR5rev:GFP-negative protoplasts in the $50 \mu \mathrm{M}$ yucasin condition. In 889 b and c, each row shows cell diameter (left panel) and change in cell diameter comparted to Day 0 890 (right panel) for individual DR5rev:GFP protoplasts.

892 Supplementary Figure 7 Roles of histone acetylation, auxin biosynthesis and exogenous 2,4-D 893 in regulating expression of cell cycle genes during protoplast reprogramming. a, Heatmap 894 representing the transcriptional changes for 172 genes specifically expressed at S phase and 185 895 genes specifically expressed at G2/M phase. The left three columns show expression levels in the 896 control, $50 \mu \mathrm{M}$ yucasin, and $50 \mu \mathrm{M}$ MB-3 condition as values normalized $\left(\log _{2} \mathrm{FC}\right)$ to Day 0 . The 897 'Relative levels to control' columns show the normalized expression levels in the yucasin or MB-3 898 condition compared to the control condition for respective time points as relative values $\left(\log _{2} \mathrm{FC}\right)$.

899 Average RPM indicates the overall expression level for each gene. Gene sets are taken from 900 Kobayashi et al. ${ }^{29}$. Two genes in the original gene set were omitted from the heatmap of S phase 901 genes since they were not expressed in all samples (see Supplementary Table 7b). b, Heatmap 902 representing the transcriptional changes for the same gene sets as in $\mathbf{a}$. The left three columns show 903 expression levels at Day 4 in the control, $50 \mu \mathrm{M}$ yucasin, and 2,4-D-omitted (named '- 2,4-D')

904 condition as values normalized $\left(\log _{2} \mathrm{FC}\right)$ to Day 0 . The 'Relative levels to control' columns show the 905 normalized expression levels in yucasin or - 2,4-D condition compared to the control condition for 906 respective time points as relative values $\left(\log _{2} \mathrm{FC}\right)$. Two genes in the original gene set were omitted 907 from the heatmap of S phase genes since they were not expressed in all samples (see Supplementary 908 Table 7d). 
910 Supplementary Figure 8 Roles of histone acetylation and auxin biosynthesis in regulating 911 expression of chloroplast-related genes during protoplast reprogramming. Heatmap 912 representing the transcriptional changes for genes implicated in chloroplast biogenesis and function 913 in yucasin or MB-3-treated protoplasts. Genes are listed in the same order as in Supplementary Fig. 9143.

916 Supplementary Figure 9 Roles of ARF7, ARF19, IAA3 and IAA18 in protoplast cell cycle

917 reinitiation. a, Callus formation efficiency of protoplasts isolated from WT, arf7 and arf19 mutants.

918 Error bars represent standard error. $\mathrm{n}=30$ from 6 biological replicates for the WT and 15 from 3 919 biological replicates for the others. ${ }^{* * *} P<0.001$ (two-tailed Student's $t$-test or Welch's $t$-test 920 compared to WT). b, Transcriptional changes for Aux/IAA genes in Arabidopsis and their protein 921 interaction with ARF7 and ARF19. The leftmost column shows heatmaps representing relative 922 expression levels $\left(\log _{2} \mathrm{FC}\right)$ in protoplasts at Day 0 compared to 23 DAS leaves. The second column 923 shows heatmaps representing relative expression levels $\left(\log _{2} \mathrm{FC}\right)$ in protoplasts at Day 2, 4, 6 and 14 924 compared to Day 0. Average RPM indicates the overall expression level for each gene in 23 DAS 925 leaves or cultured protoplasts. Right column shows Aux/IAA proteins that can interact with ARF7 926 and/or ARF19 based on Piya et $a .^{42}$. The strength of protein interaction is shown in gray and black 927 circles.

929 Supplementary Figure 10 Roles of LBD16, LBD18 and LBD33 in callus formation from 930 hypocotyl explants. Hypocotyl explants from WT and lbd16-1 lbd18-1 lbd33-1 seedlings were 931 incubated on CIM for 21 days. Callus formation was assessed using more than 80 explants from 2 932 biological replicates for each genotype and phenotypic reproducibility was confirmed. Scale bars are $9331 \mathrm{~mm}$. 
938 Supplementary Table 2 | Expression data for chloroplast-related genes. Expression data of the 939 chloroplast-related genes listed in Supplementary Fig. 3 and Supplementary Fig. 8 at Day 0 and 940 Day 14 in control or MB-3 conditions (Experiment A) and at Day 0, Day 2, Day 4 and Day 6 in the

941 control, yucasin and MB-3 conditions (Experiment B). The 'logFC' columns show the relative 942 expression levels $\left(\log _{2} \mathrm{FC}\right)$ at each time point compared to intact leaves (in the case of Day 0 data) or 943 to Day 0 (in other cases).

945 Supplementary Table 3 | Lists of the differentially expressed genes shown in Fig. 2c. a, 4,679 946 genes that are significantly upregulated in the control condition at Day 14 compared to Day 0. b, 9474,287 genes that are significantly upregulated in the MB-3 condition at Day 14 compared to Day 0. c,

9481,186 genes that show significantly higher expression levels in the control condition compared to the 949 MB-3 condition at Day 14. d, 395 genes that are that significantly upregulated only in the control 950 condition but not in the MB-3 condition at Day 14 compared to Day 0 and also have significantly 951 higher expression levels in the control condition than in the MB-3 condition at Day 14. e, 140 genes 952 that are that significantly upregulated in both the control and MB-3 conditions at Day 14 from Day 0, 953 but with significantly higher expression levels in the control condition than in the MB-3 condition at 954 Day 14.

956 Supplementary Table $4 \mid$ The top 50 categories from GO enrichment analysis for the 535 957 upregulated genes. GO categories classified as biological functions were used for the analysis.

959 Supplementary Table 5| A list of genes involved in biosynthesis and metabolism of 960 indole-containing compounds that show significantly stronger upregulation in the control 961 condition compared to the MB-3 condition. a, The genes categorized under GO terms related to 
indole-containing compound biosynthetic or metabolic process based on the analysis presented in

963 Supplementary Table 4. The color key is the same as Fig. 2d. b, Expression data for a. of the genes involved in IAA biosynthesis and metabolism in intact leaves and in protoplasts at Day

9670 and Day 14. Gene sets were selected based on annotations at Kyoto Encyclopedia of Genes and 968 Genomes (KEGG) (https://www.genome.jp/kegg/kegg_ja.html) and The Arabidopsis Information 969 Resource (TAIR) (https://www.arabidopsis.org/index.jsp). b, The expression of representative IAA 970 biosynthetic genes at Day 0, Day 2, Day 4 and Day 6.

972 Supplementary Table 7| Expression data for cell cycle genes during the first week of 973 protoplast culture. a, The expression of the core cell cycle genes listed in Fig. 4a. b, The expression 974 of the genes specifically expressed in $\mathrm{S}$ phase. The genes are listed in the same order as in 975 Supplementary Fig. 7a. c, The expression of the genes specifically expressed in G2/M phase. The 976 genes are listed in the same order as in Supplementary Fig. 7a. d, The expression of the genes 977 specifically expressed in S phase. The genes are listed in the same order as in Supplementary Fig. 978 7b. e, The expression of the genes specifically expressed in G2/M phase. The genes are listed in the 979 same order as in Supplementary Fig. 7b.

981 Supplementary Table $8 \mid$ Expression data for the genes coding auxin signalling components 982 that contribute to callus formation in tissue culture.

984 Supplementary Table 9 | Expression data for Aux/IAA genes in leaves and protoplasts. 985

986 Supplementary Table 10| Primers used in this study. 
987 The primer sequences used for YUC1 CDS (coding sequence) cloning and for YUC1 RT-qPCR are 988 from Sugawara et al. ${ }^{70}$. The primer sequences for PP2A3 RT-qPCR are from Rymen et al. ${ }^{69}$. 989 
a

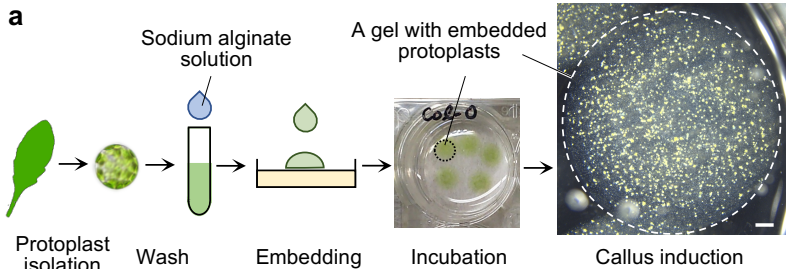

Embedding

Incubation

Callus induction

C
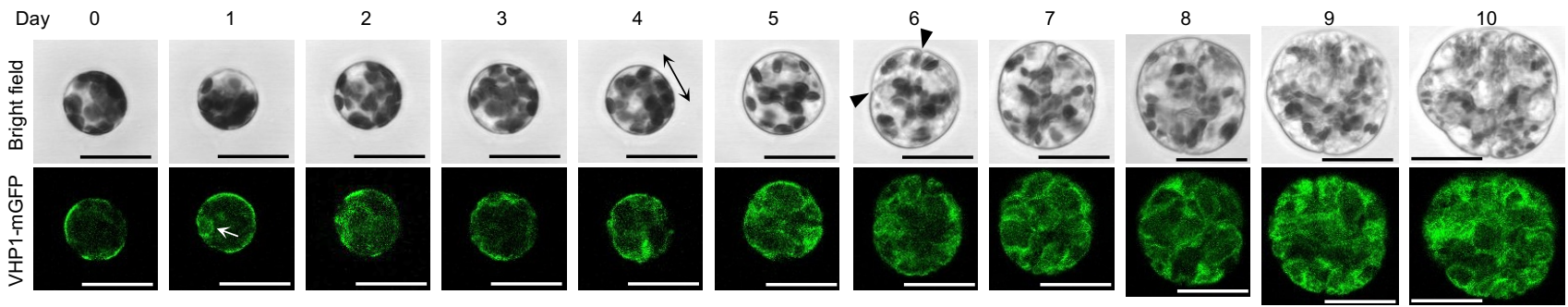

Figure 1 Leaf mesophyll protoplasts reprogram into a pluripotent state and regenerate shoots in vitro. a, Diagram showing the key steps for protoplast isolation and callus induction. The circles with dashed lines indicate a sodium alginate gel that contains protoplasts. $\mathbf{b}$, Light microscopy images of a freshly isolated leaf mesophyll protoplast (1), a protoplast that has undergone the first cell division (2), callus formed from a protoplast (3 to 5), and new shoots formed from protoplast-derived callus $(6,7)$. c, Time-lapse confocal microscopy images of a leaf mesophyll protoplast undergoing cellular reprogramming from Day 0 to Day 10. Vacuolar morphology is visualized by VHP1-mGFP. The double-headed arrow indicates the direction of cell elongation and arrowheads mark the plane of initial cell division. The white arrow highlights the initial appearance of vacuolar strand-like structures. Scale bars are $10 \mu \mathrm{m}$ (1 to 3 in a), $100 \mu \mathrm{m}(\mathbf{4}$ to 6 in a), $1 \mathrm{~mm}(\mathbf{b})$ and $30 \mu \mathrm{m}$ (c). 


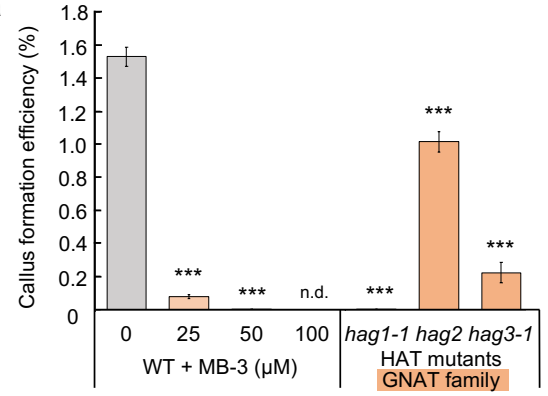

c

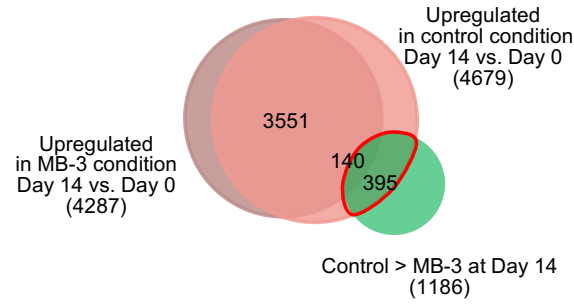

f

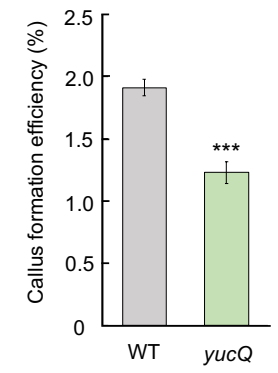

g

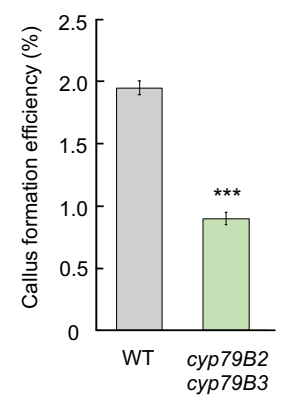

b
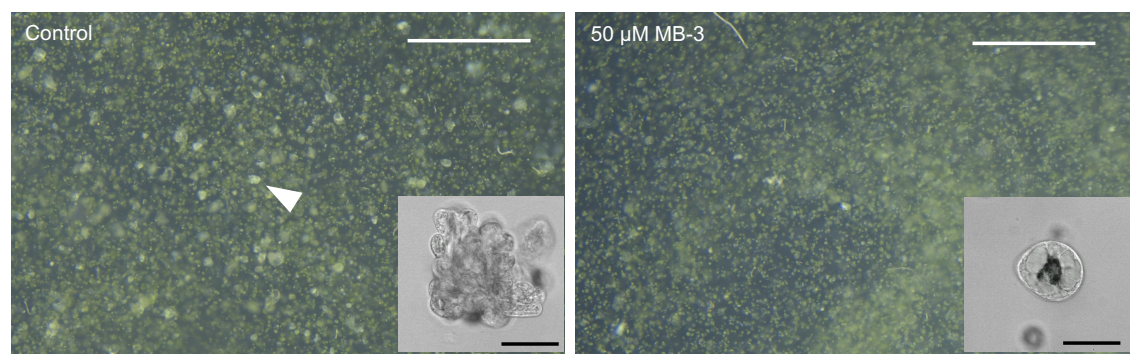

d

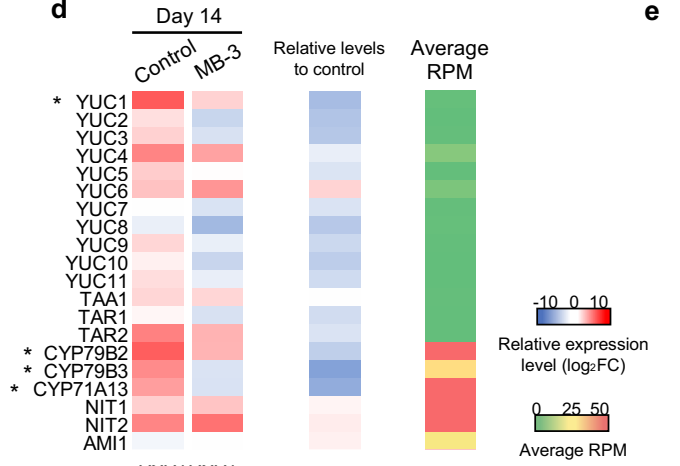

h

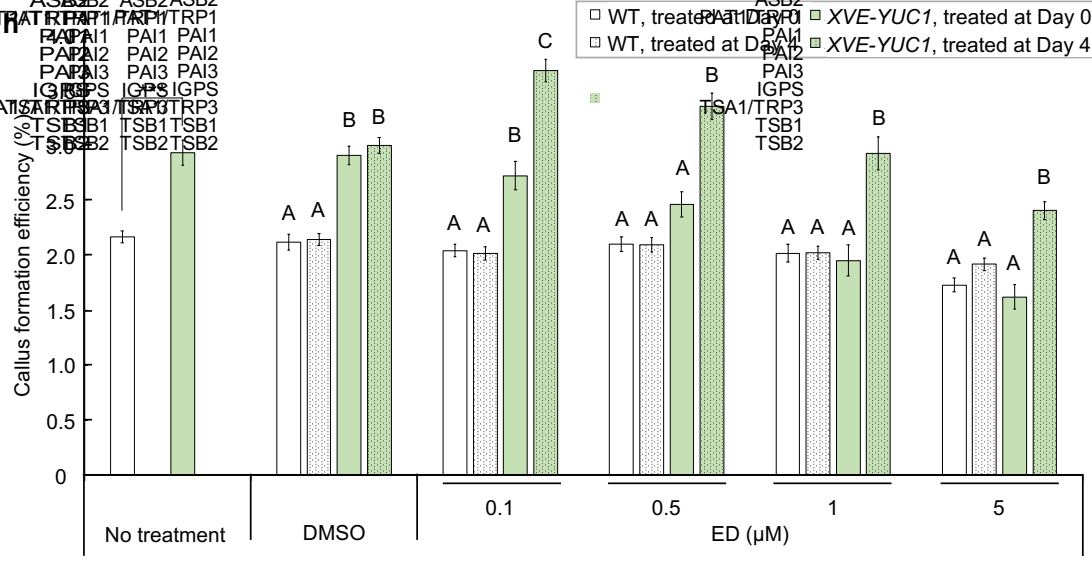

Figure 2 Histone acetylation is required to reinitiate cell division in leaf mesophyll protoplasts. a, Callus formation efficiency of MB3-treated WT, hag1-1, hag2 and hag3-1 protoplasts at Day 14. MB-3 was added at the beginning of culture (Day 0). Error bars represent standard error. $\mathrm{n}=40$ from 8 biological replicates for the WT control, 15 from 3 biological replicates for hagl-1 and hag2, and 20 from 4 biological replicates for all others. *** $P<0.001$ (two-tailed Welch's $t$-test compared to WT control). n.d. not determined. b. Bright field images of WT protoplasts incubated in control (left panel) and 50 $\mu \mathrm{M}$ MB-3 condition (right panel) for 14 days. The white arrowhead indicates a single callus. The bottom right insets show confocal microscopy images of callus cells (left panel) and an undivided protoplast (right panel). c, A Venn diagram of genes significantly upregulated in control and $50 \mu \mathrm{M}$ MB-3 conditions. The green circle indicates genes that show significantly higher expression in the control compared to the MB-3 condition at Day 14 . Numbers in brackets show total numbers of genes included in each group. The red outline highlights 535 genes that show significantly stronger upregulation in the control compared to the MB-3 condition. d, Heat map of the transcriptional changes for representative genes implicated in IAA biosynthesis. The left two columns show the expression levels in the control and MB-3 conditions at Day 14 as values normalized $\left(\log _{2}\right.$ FC) to Day 0 . The 'Relative levels to control' column shows the differential expression levels in the MB-3 condition compared to the control at Day 14 as relative values (log $\mathrm{FC}_{2}$ ). Average reads per million (RPM) indicates the overall expression level for each gene. Asterisks mark the genes that are included from the 535 upregulated genes in c. e, Callus formation efficiency of yucasin-treated WT protoplasts. Error bars represent standard error. $\mathrm{n}=30$ from 6 biological replicates for the WT control and 15 from 3 biological replicates for the others. ${ }^{* *} P<0.001$ (two-tailed Student's $t$-test or Welch's $t$-test compared to WT control). n.d. not determined. f, Callus formation efficiency of WT and yuc 3 yuc 5 yuc7 yuc 8 yuc 9 (yucQ) protoplasts. Error bars represent standard error. $\mathrm{n}=15$ from 3 biological replicates. ${ }^{* * *} P<0.001$ (two-tailed Student's $t$-test compared to WT). g, Callus formation efficiency of WT and cyp79B2 cyp $79 B 3$ protoplasts. Error bars represent standard error. $\mathrm{n}=15$ from 3 biological replicates. ${ }^{* * *} P<0.001$ (two-tailed Student's $t$-test compared to WT). h, Callus formation efficiency of $X V E$-YUC1 protoplasts. DMSO or $\beta$-estradiol (ED) were added to PCIM at the indicated final concentration at the beginning (Day 0) or Day 4 of culture. Error bars represent standard error. $\mathrm{n}=15$ from 3 biological replicates. For 'No treatment', $* * * P<0.001$ (two-tailed Welch's $t$-test compared to WT under the same treatment). For the others, different letters indicate significant differences based on one-way ANOVA with post hoc Tukey's HSD test $(P<0.001)$ within each treatment group. Scale bars are $1 \mathrm{~mm}$ (b) and $50 \mu \mathrm{m}$ (insets in $\mathbf{b}$ ). 

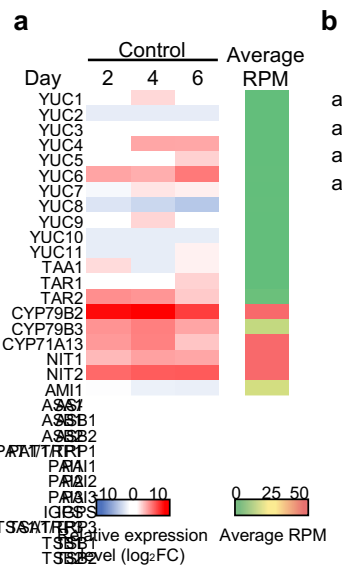

b
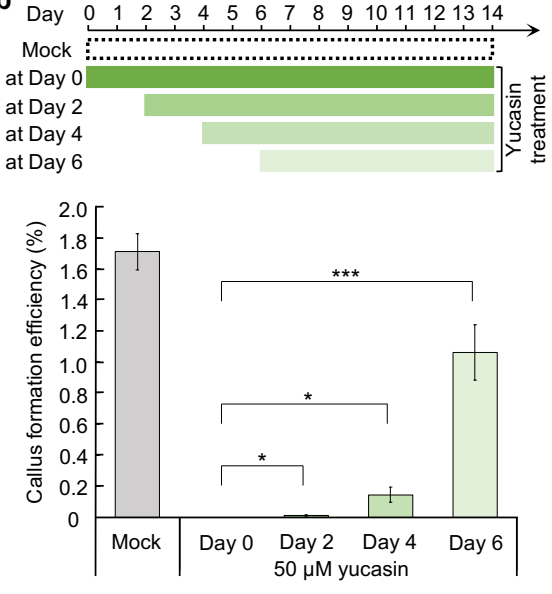
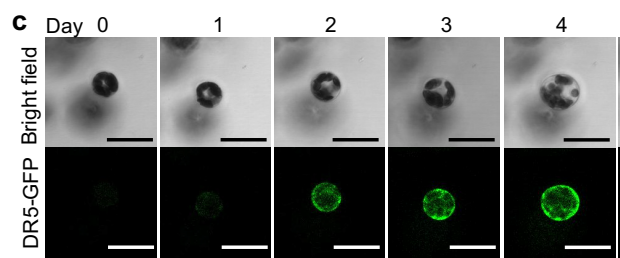

5 6

d

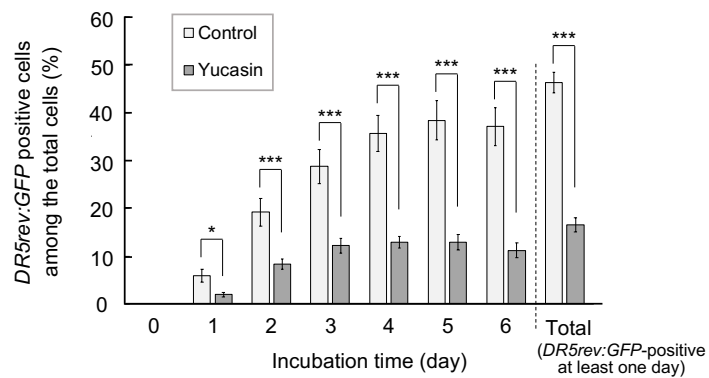

Figure 3 Early auxin biosynthesis is required to reinitiate cell division in leaf mesophyll protoplasts. a, Heat map of the transcriptional changes for representative IAA biosynthesis genes at Day 2, 4 and 6 in the control condition. Values $\left(\log _{2} \mathrm{FC}\right)$ are relative to Day 0 . Average RPM indicates the overall expression level for each gene. b, Callus formation efficiency of WT protoplasts treated with $50 \mu \mathrm{M}$ yucasin at different time points. Diagram shows the timing of yucasin treatment. Error bars represent standard error. $\mathrm{n}=15$ from 3 biological replicates. $* P<0.05, * * * P<0.001$ (two-tailed Welch's $t$-test compared to yucasin treatment at Day 0). c, Time-lapse confocal microscopy images of a mesophyll protoplast that undergoes cell division at Day 5. Auxin response is visualized by DR5rev:GFP expression. d, Frequency of DR5rev:GFP-expressing (positive) protoplasts in the control or $50 \mu \mathrm{M}$ yucasin condition among all tested DR5rev:GFP protoplasts. Error bars represent standard error. $\mathrm{n}=9$ for control and 7 for yucasin-treated protoplasts from 2 biological replicates. ${ }^{* * *} P<0.001$ (two-tailed Student's $t$-test or Welch's $t$-test). Scale bars are $30 \mu \mathrm{m}(\mathbf{c})$. 


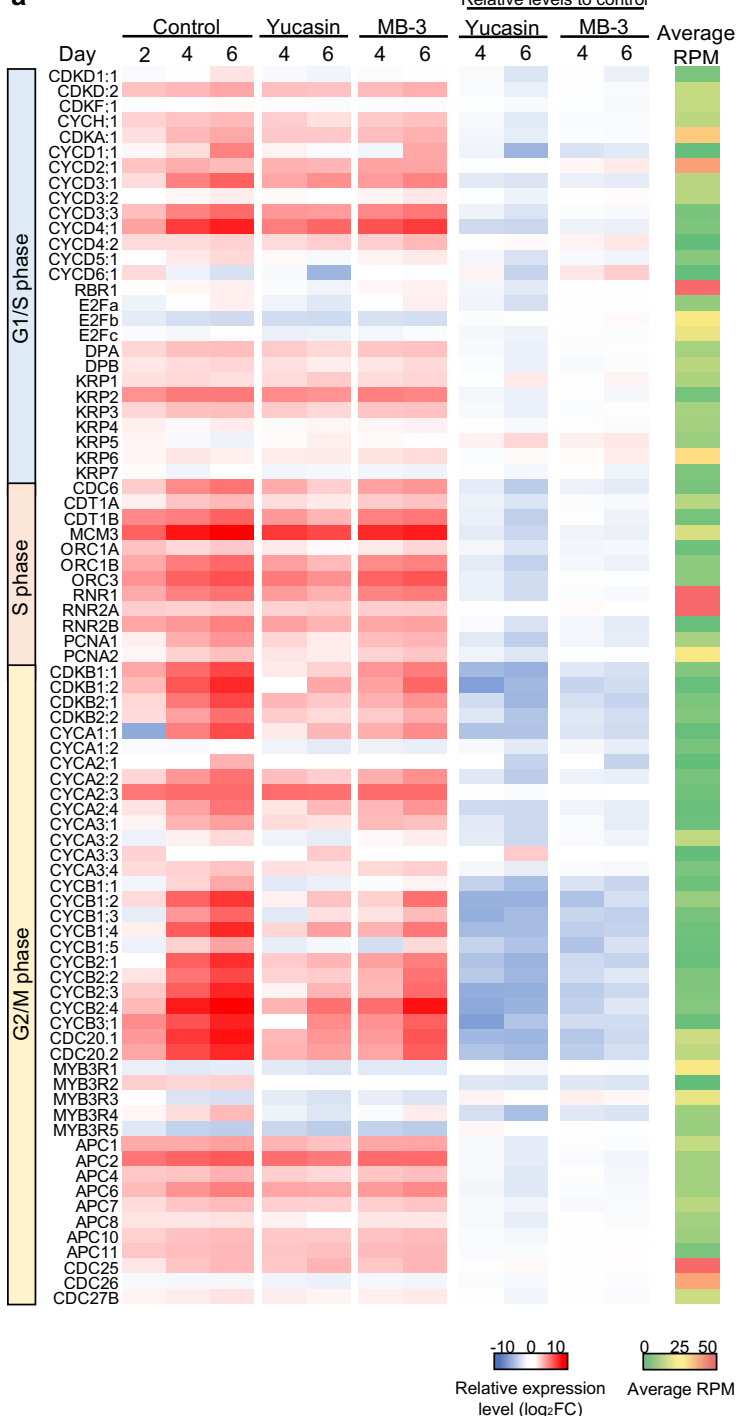

b

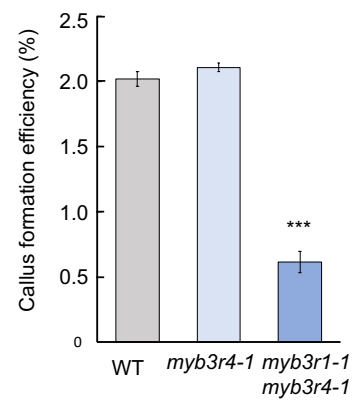

C

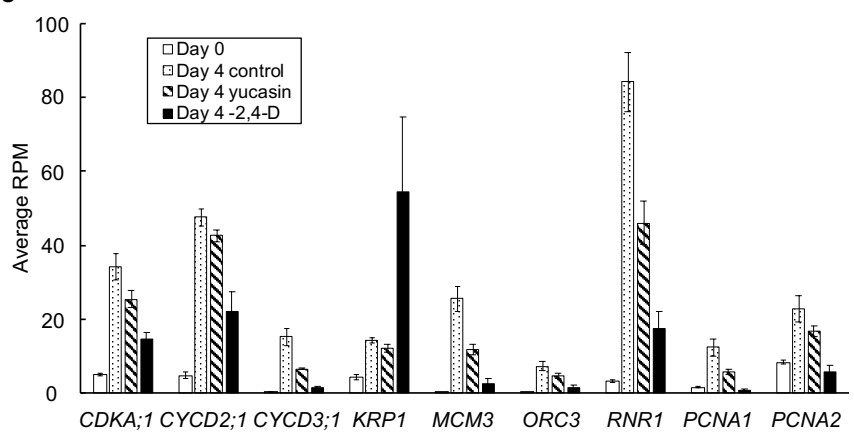

Figure 4 Auxin biosynthesis is required to transcriptionally activate the G2/M phase genes through MYB3R1 and MYB3R4. a, Heat map representing the transcriptional changes for genes encoding core cell cycle regulators at G1/S, S and G2/M phases at Day 2, 4 and 6. The left three columns show expression levels in the control, $50 \mu \mathrm{M}$ yucasin and $50 \mu \mathrm{M}$ MB-3 conditions as values normalized $\left(\log _{2} \mathrm{FC}\right)$ to Day 0. The 'Relative levels to control' columns show the normalized expression levels in the yucasin or MB-3 condition compared to the control condition for respective time points as relative values $\left(\log _{2} \mathrm{FC}\right)$. Average RPM indicates the overall expression level for each gene. Gene sets are selected based on Kalve et al. ${ }^{47}$. b, Callus formation efficiency of WT, myb3r4-1 and myb3r1-1 myb3r4-1 protoplasts. Error bars represent standard error. $\mathrm{n}=20$ from 4 biological replicates for the WT and myb3r1-1 myb3r4-1 and $\mathrm{n}=15$ from 3 biological replicates for myb3r4-1. ${ }^{* * *} P<0.001$ (two-tailed Student's $t$-test compared to WT). c, Examples of the G1/S phase-related genes that are differently expressed in the - 2,4-D condition compared to the control and yucasin conditions at Day 4. Error bars represent standard error. $n=6$ for Day 0 and the control condition at Day4 and $n=3$ for the yucasin and - 2,4-D conditions at Day 4. 


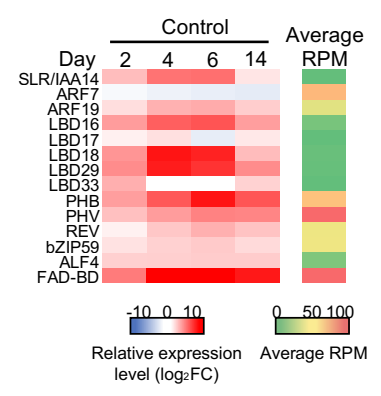

C

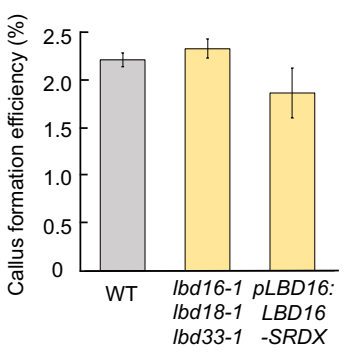

b

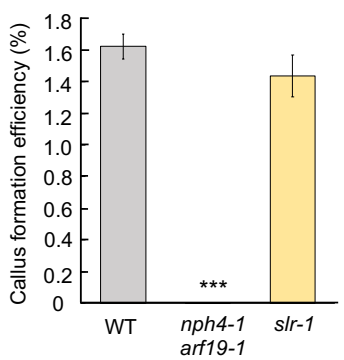

d

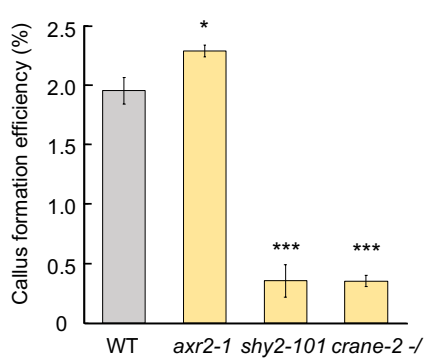

Figure 5 | ARF7/ARF19 and IAA3/IAA18-mediated auxin signalling pathway drives cell cycle reinitiation in leaf mesophyll protoplasts. a, Heat map representing the transcriptional changes for genes implicated in auxin-induced callus formation in tissue culture. Expression levels at Day 2, 4 and 6 in the control condition are shown as values normalized $\left(\log _{2} \mathrm{FC}\right)$ to Day 0. Average RPM indicates the overall expression level for each gene. Gene sets are taken from Ikeuchi et al. ${ }^{6}$. b. Callus formation efficiency of WT, nph4-1 arf19-1 and slr-1 protoplasts. Error bars represent standard error. $\mathrm{n}=25$ from 5 biological replicates for the WT and 20 from 4 biological replicates for the others. ${ }^{* *} P<0.001$ (two-tailed Student's $t$ test or Welch's $t$-test compared to WT). c, Callus formation efficiency of WT, $l b d 16-1 l b d 18-1 l b d 33-1$ and $p L B D 16: L B D 16-S R D X$ protoplasts. Error bars represent standard error. $\mathrm{n}=20$ from 4 biological replicates for the WT and 15 from 3 biological replicates for the others. No statistical difference was detected (two-tailed Student's $t$-test or Welch's $t$-test compared to WT). d, Callus formation efficiency of WT, shy2-101, axr2-1 and crane-2 protoplasts. Since heterozygous and homozygous crane-2 are morphologically indistinguishable, both genotypes were mixed for protoplast preparation. Error bars represent standard error. $\mathrm{n}=25$ from 5 biological replicates for the WT and 15 from 3 biological replicates for the others. $* P<0.05, * * * P<0.001$ (two-tailed Student's $t$-test or Welch's $t$ test compared to WT). 
<smiles></smiles>

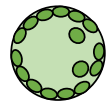
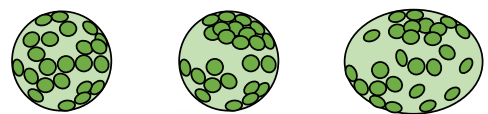

S

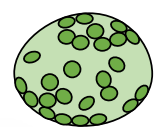

G2
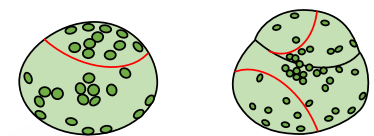

M
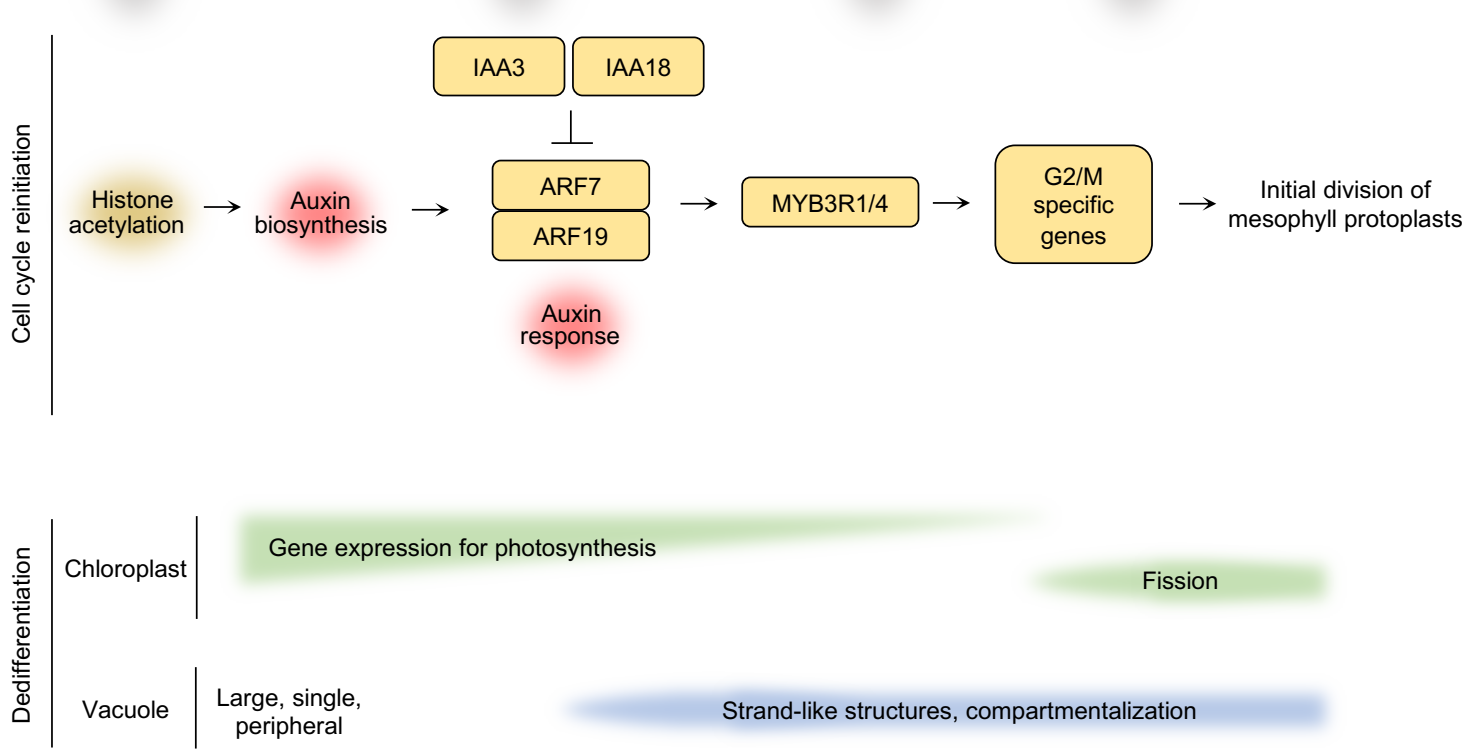

Figure 6 | Hypothetical model describing the molecular mechanism of developmental reprogramming in leaf mesophyll protoplasts. Freshly isolated Arabidopsis leaf mesophyll protoplasts undergo dramatic developmental reprogramming and produce pluripotent callus when cultured in the presence of auxin and cytokinin. Histone acetylation is required to reinitiate the mitotic cell cycle and one of the key downstream pathways regulated by histone acetylation is the transcription of auxin biosynthesis genes. Endogenously produced auxin, IAA, in turn activates auxin response in protoplasts, possibly in an ARF7/ARF19 and IAA3/IAA18-dependent manner. Endogenous auxin is responsible for the transcriptional upregulation of G2/M genes to complete the initial cell division, and such cell cycle regulation likely involves auxin-dependent induction of MYB3R4. MYB3R4 and MYB3R1 might be also regulated at the post-translational level. Another key feature of developmental reprogramming that protoplasts undergo at an early stage of culture is the cellular dedifferentiation, which is marked, for instance, by drastic downregulation of photosynthetic genes and upregulation of chloroplast fission genes. These transcriptional changes are independent of histone acetylation and auxin biosynthesis. In addition, a large, single vacuole, typical of differentiated cells, becomes compartmentalized and starts to resemble what is found in proliferating or elongating cells. 